\title{
Resolved, expanding jets in the Galactic black hole candidate XTE J1908+094
}

\author{
A. P. Rushton, ${ }^{1,2 \star ~ J . ~ C . ~ A . ~ M i l l e r-J o n e s, ~}{ }^{3}$ P. A. Curran,${ }^{3} \dagger$ G. R. Sivakoff, ${ }^{4}$ \\ M. P. Rupen, ${ }^{5}$ Z. Paragi, ${ }^{6}$ R. E. Spencer, ${ }^{7}$ J. Yang, ${ }^{8,9}$ D. Altamirano, ${ }^{2}$ \\ T. Belloni, ${ }^{10}$ R. P. Fender, ${ }^{1}$ H. A. Krimm, ${ }^{11,12}$ D. Maitra, ${ }^{13}$ S. Migliari, ${ }^{14,15}$ \\ D. M. Russell, ${ }^{16}$ T. D. Russell, ${ }^{17,3}$ R. Soria ${ }^{3,18}$ and V. Tudose ${ }^{19}$ \\ ${ }^{1}$ Department of Physics, Astrophysics, University of Oxford, Keble Road, Oxford OX1 3RH, UK \\ 2 School of Physics and Astronomy, University of Southampton, Highfield, Southampton SO17 1BJ, UK \\ ${ }^{3}$ International Centre for Radio Astronomy Research - Curtin University, GPO Box U1987, Perth, WA 6845, Australia \\ ${ }^{4}$ Department of Physics, University of Alberta, 4-181 CCIS, Edmonton, AB T6G 2E1, Canada \\ 5 National Research Council of Canada, Herzberg Astronomy and Astrophysics, Dominion Radio Astrophysical Observatory, \\ PO Box 248, Penticton, BC V2A 6J9, Canada \\ 6 Joint Institute for VLBI ERIC (JIVE), Postbus 2, NL-7990 AA Dwingeloo, the Netherlands \\ 7 Jodrell Bank Observatory, University of Manchester, Macclesfield, Cheshire SK11 9DL, UK \\ 8 Department of Earth and Space Sciences, Chalmers University of Technology, Onsala Space Observatory, SE-439 92 Onsala, Sweden \\ ${ }^{9}$ Shanghai Astronomical Observatory, Chinese Academy of Sciences, 200030 Shanghai, P. R. China \\ 10 Istituto Nazionale di Astrofisica, Osservatorio Astronomico di Brera, Via E. Bianchi 46, I-23807 Merate, Italy \\ 11 Universities Space Research Association, 7178 Columbia Gateway Dr, Columbia, MD 21046, USA \\ 12 National Science Foundation, 4201 Wilson Blvd, Arlington, VA 22230, USA \\ 13 Department of Physics 83 Astronomy, Wheaton College, Norton, MA 02766, USA \\ 14 European Space Astronomy Centre (ESAC), 28692 Villanueva de la Cañada, Madrid, Spain \\ 15 Department of Quantum Physics and Astrophysics 83 Institute of Cosmos Sciences, University of Barcelona, 08028 Barcelona, Spain \\ 16 New York University Abu Dhabi, PO Box 129188, Abu Dhabi, UAE \\ 17 Anton Pannekoek Institute for Astronomy, University of Amsterdam, PO Box 94249, NL-1090 GE Amsterdam, the Netherlands \\ 18 Sydney Institute for Astronomy, School of Physics A28, The University of Sydney, NSW 2006, Australia \\ 19 Institute for Space Sciences, Atomistilor 409, PO Box MG-23, 077125 Bucharest-Magurele, Romania
}

Accepted 2017 February 27. Received 2017 February 26 ; in original form 2017 January 16

\begin{abstract}
Black hole X-ray binaries undergo occasional outbursts caused by changing inner accretion flows. Here we report high-angular resolution radio observations of the 2013 outburst of the black hole candidate X-ray binary system XTE J1908+094, using data from the VLBA and EVN. We show that following a hard-to-soft state transition, we detect moving jet knots that appear asymmetric in morphology and brightness, and expand to become laterally resolved as they move away from the core, along an axis aligned approximately $-11^{\circ}$ east of north. We initially see only the southern component, whose evolution gives rise to a $15-\mathrm{mJy}$ radio flare and generates the observed radio polarization. This fades and becomes resolved out after 4 days, after which a second component appears to the north, moving in the opposite direction. From the timing of the appearance of the knots relative to the X-ray state transition, a $90^{\circ}$ swing of the inferred magnetic field orientation, the asymmetric appearance of the knots, their complex and evolving morphology, and their low speeds, we interpret the knots as working surfaces where the jets impact the surrounding medium. This would imply a substantially denser environment surrounding XTE J1908+094 than has been inferred to exist around the microquasar sources GRS 1915+105 and GRO J1655-40.
\end{abstract}

Key words: X-rays: binaries - ISM: jets and outflows - Radio continuum: stars Stars: individual: XTE J1908+094 


\section{INTRODUCTION}

Galactic black hole X-ray binaries (BH XRBs) exhibit a plethora of accretion 'states' (e.g. Belloni 2010), allowing us to study the different regimes in which a black hole accretes matter and interacts with the surrounding environment. Over relatively short time scales, the spectral energy distribution of a $\mathrm{BH}$ XRB can change significantly due to variations in the structure and geometry of the accretion flow. BH XRBs also drive extended outflows (or jets), whose structure appears to be directly related to these changes in the inner accretion flow. During accretion states that are dominated by hard, non-thermal X-ray emission, an AUscale, compact, quasi-steady jet can be observed, whereas transitions from the hard state to softer X-ray states are associated with the ejection of relativistically-moving 'knots' of plasma that are no longer causally connected to the accretion flow (as reviewed in, e.g. Fender 2006). While the structure and composition of the jets are not known, Gallo et al. (2005), Russell et al. (2007) and Sell et al. (2015) have shown that the power of the outflows can be comparable to the total bolometric luminosity of the binary system.

Perhaps the most famous jet-producing black hole is GRS 1915+105, which was the first Galactic source observed to show superluminal motion (e.g. Mirabel \& Rodríguez 1994; Fender et al. 1999). The source has shown repeated major radio outbursts, in which discrete knots of optically-thin plasma are directly resolved by high angular resolution monitoring whenever a radio flare occurs during the plateau state (Rushton et al. 2010). Although transient radio ejecta have been best studied in GRS 1915+105, there are a handful of other systems that have shown discrete, resolved jet knots on milliarcsecond scales, including GRO J1655-40 (Tingay et al. 1995; Hjellming \& Rupen 1995), Cyg X-3 (Mioduszewski et al. 2001; Miller-Jones et al. 2004), H1743-322 (Miller-Jones et al. 2012), and XTE J1752-223 (Yang et al. 2010). These sources all appear to display resolved jets following a hard-to-soft X-ray state change, with typical proper motions of 10-100 mas $\mathrm{d}^{-1}$ implying velocities $v \sim 0.1-0.9 \mathrm{c}$ (for distances of $1-10 \mathrm{kpc}$ Ling et al. 2009; Reid et al. 2014). Some of these sources have shown evidence for deceleration of the knots (e.g. Corbel et al. 2002, 2005; Miller-Jones et al. 2011; Yang et al. 2011), implying that an external shock is produced as the jets interact with the interstellar medium (ISM) (Corbel et al. 2002).

Most of the individual jet ejecta from BH XRBs have remained unresolved perpendicular to the jet axis, showing no lateral expansion (Miller-Jones et al. 2006) or interaction with the ISM. One of the few Galactic cases in which the jet/ISM interaction has been directly resolved is the (persistent) neutron star XRB Sco X-1. Fomalont et al. (2001) analysed the expansion of two radio lobes from Sco $\mathrm{X}-1$, which reached a minimum resolved size of $4 \times 10^{8} \mathrm{~km}$ ( $\sim 1$ mas) over a period of a few hours. They argued that the lobes must consist of electrons adiabatically expanding in a working surface, akin to the hot spots found in many extragalactic radio sources. Another case where lateral expansion of the jet ejecta has been directly resolved is the BH XRB XTE J1752-223, for which an expansion speed of $0.9 \pm 0.1$ mas d $^{-1}$ (i.e. $0.05 c[d / 10 \mathrm{kpc}]$ ) was measured in one of the decelerating jet components, again interpreted as interaction with the ISM (Yang et al. 2010).

A basic phenomenological explanation for the jet behaviour (Fender et al. 2004) couples increased mass accretion rate to an increasingly powerful compact jet, until a rapid increase of the Lorentz factor causes internal shocks to form within the jets (Kaiser et al. 2000), producing the observed discrete knots of plasma. Models show that shell collisions can give rise to internal shocks, which act as an electron re-energization mechanism in an adiabatic conical jet (e.g. Kaiser 2006; Jamil et al. 2010).

While internal shocks can temporarily illuminate jets, more continuous, steady jets in microquasars should eventually terminate in strong shocks and could inflate radio lobes (e.g. Mirabel et al. 1992; Rodriguez et al. 1992; Pakull et al. 2010) similar to extragalactic jet sources. However, Heinz (2002) argued that the direct detection of radio lobes from $\mathrm{X}$-ray binaries is difficult due to the their low surface brightness, whereas indirect detection (e.g. X-ray hot spots) is common. Based on how far the jet ejecta propagated downstream before decelerating, they estimated the densities of the media surrounding GRS 1915+105 and GRO J1655-40 to be $n \leq 10^{-3} \mathrm{~cm}^{-3}$, implying previous outbursts could have evacuated low density bubbles around these binaries. However, they also argued that hotspots can suddenly appear once the ejecta reach the boundary of the radio lobe and collide with the denser ISM.

A further complication can arise if the binary has a high velocity due to a natal supernova kick. In this case, the interaction of the jets with the ISM could then lead to the production of asymmetric trails and bow shocks (Heinz et al. 2008; Wiersema et al. 2009; Yoon et al. 2011).

\section{$1.1 \quad$ XTE J1908+094}

XTE J1908+094 was serendipitously discovered by the Proportional Counter Array (PCA) on board the Rossi X-ray Timing Explorer (RXTE), and by BeppoSAX, during an outburst in 2002 (Woods et al. 2002; Feroci et al. 2002). The $\mathrm{X}$-ray spectral evolution was studied in detail by in't Zand et al. (2002b) and Göğüş et al. (2004), who found that after a rising hard state phase, the source underwent a hard-to-soft spectral state transition typical of $\mathrm{BH}$ XRBs. It remained in the soft state for $58 \mathrm{~d}$ before transitioning back to the hard state and decaying. A second X-ray outburst took place approximately a year after the first, peaking in January 2003.

A radio counterpart to the original 2002 outburst was first discovered in March 2002 by the Very Large Array (Rupen et al. 2002a), and remained active for 2-3 months (Rupen et al. 2002b). A near-infrared (NIR) counterpart was initially reported by Chaty et al. (2002) using the European Southern Observatory's New Technology Telescope (NTT), and was later resolved into two separate objects (Chaty et al. 2006), whose properties were used to conclude that the black hole candidate system is indeed a lowmass X-ray binary. During the subsequent decay of the outburst, Jonker et al. (2004) investigated the disc-jet coupling with (near-)simultaneous radio and X-ray observations of XTE J1908+094 using the VLA, Westerbork Synthesis Radio Telescope (WSRT) and Chandra.

The source distance is poorly constrained, although in't Zand et al. (2002b) used the peak bolometric flux of the 
2002 outburst to place a lower limit of $>6.8 \mathrm{kpc}$. Using the measured flux of the soft-to-hard X-ray state transition, which is known to occur at $1-3 \%$ of the Eddington luminosity (Maccarone 2003; Kalemci et al. 2013), Curran et al. (2015) found a distance range of 4.8-13.6 kpc (for black hole masses of $\left.3-10 M_{\odot}\right)$. Throughout this paper we therefore assume a canonical distance of $8 \mathrm{kpc}$.

A new outburst from XTE J1908+094 was detected in late 2013 by Swift/BAT (Krimm et al. 2013a), triggering a multi-wavelength observing campaign. The $15-50 \mathrm{keV} \mathrm{X}-$ ray flux observed with the Swift/BAT hard X-ray transient monitor was observed to increase from 2013 October 26 (MJD 56591), reaching $\sim 60 \mathrm{mCrab}$ by October 28 . The detection of hard X-rays triggered pointed observations by the XRT instrument on board Swift, which on October 29 (MJD 56594.84 ) found an unabsorbed flux of $1.5 \pm 0.1 \times 10^{-9} \mathrm{erg}$ $\mathrm{s}^{-1} \mathrm{~cm}^{-2}$ in the $0.3-10 \mathrm{keV}$ band, and a power-law spectrum with $\Gamma=1.63 \pm 0.07$, confirming that the source was in a hard X-ray spectral state (Krimm et al. 2013b). The hard X-ray flux measured by Swift/BAT peaked at $\sim 120 \mathrm{mCrab}$ on MJD 56595 and subsequently decreased. The following two Swift/XRT observations (taken on November 1 and 3; MJD 56597.92 and 56599.53) showed the source to be in an intermediate state, with X-ray spectra still dominated by the power-law component but with an increasing contribution from the disc blackbody. The X-ray power-law index was also seen to soften over this period, from $\Gamma=1.57$ on October 29 to $\Gamma=2.30$ by November 3 (Zhang et al. 2015). By the time of the fourth Swift/XRT observation on November 8 (MJD 56604.86), the source was in a soft X-ray spectral state, where it stayed until it became Sunconstrained in early December. Zhang et al. (2015) also found evidence for a non-constant inner disc radius during the soft state, and a disc luminosity that varied with temperature as $L \propto T^{2}$ rather than the $L \propto T^{4}$ expected from a standard Shakura-Sunyaev thin disc. They interpreted this as evidence of an optically-thick, advection-dominated slim disk (the "apparently standard" state of Kubota, Makishima \& Ebisawa 2001).

In addition to the Swift/XRT observations, both Zhang et al. (2015) and Tao et al. (2015) analyzed a set of $N u S$ TAR observations taken on November 8-9 (MJD 56604.76$56605.90)$ during the soft X-ray spectral state. They detected $\mathrm{a} \sim 40$-ks flare during which the X-ray emission increased by up to $40 \%$, driven by an increase in the power-law component of the spectrum, which also showed a significant spectral softening during the flare. Both works concluded that this could be due to an ejection event, or possibly a change in the properties of the corona. While the black hole spin could not be constrained from X-ray spectral fitting in the soft state, both groups found similar inclination angles of $20-40^{\circ}$ to the line of sight, which is similar to the $45 \pm 8^{\circ}$ inferred by Miller et al. (2009) from their modelling of the relativistic disk reflection during a bright hard state.

Radio emission from the 2013 outburst of XTEJ1908+094 was first detected by Miller-Jones et al. (2013) with the Karl G. Jansky Very Large Array (VLA), and subsequently monitored by the Arcminute Microkelvin Imager Large Array (AMI-LA) (Rushton et al. 2013a,b) and the Australia Telescope Compact Array (ATCA; Coriat et al. 2013). The detectable radio flux lasted for just over 20 days. Initially, the source exhibited quasi- steady, flat spectrum radio emission at a level of $\sim 1 \mathrm{mJy}$, simultaneous with the hard X-ray rise. On November 5 (MJD 56601), AMI-LA detected a rapid radio flare to $15 \mathrm{mJy}$, which decayed over the course of a few hours. The radio emission subsequently quenched to $<0.32 \mathrm{mJy}$ on MJD 56602.7, before undergoing a much longer-duration flare that peaked at $13 \mathrm{mJy}$ on MJD 56607.1 and decayed slowly over the following ten days, becoming undetectable by MJD 56618 (Curran et al. 2015).

As BH XRB outbursts are known to be associated with resolved radio jets (Fender 2006), we triggered a series of high spatial-resolution Very Long Baseline Interferometry (VLBI) monitoring observations following the detection of the X-ray state transition. In this paper we present these high-resolution observations, taken with the Very Long Baseline Array (VLBA) and the European VLBI Network $(\mathrm{EVN})$ telescopes, which resolved the radio emission into moving, expanding jet knots. We describe the observations in Section 2, our fitting of the expansion and motion of the resolved jet structure in Section 3, and possible interpretations of the observed knots in Section 4.

\section{OBSERVATIONS AND REDUCTION}

\subsection{VLBI observations}

\subsubsection{VLBA}

Following the detection of radio emission from the 2013 outburst, we observed XTE J1908+094 over nine epochs with the Very Long Baseline Array (VLBA), spaced over $14 \mathrm{~d}$, as detailed in Table 1. At each epoch, we observed at a central frequency of $8.4 \mathrm{GHz}$, using $256 \mathrm{MHz}$ of bandwidth. We used $\mathrm{J} 1800+3848$ as a fringe finder source, and the observations were phase-referenced to the nearby $\left(0.39^{\circ}\right)$ calibrator source J1907+0907. The phase referencing cycle time was $3 \mathrm{~min}$, spending $130 \mathrm{~s}$ on the target and $50 \mathrm{~s}$ on the calibrator in each cycle. Every seventh cycle we substituted the scan on XTE J1908+094 for a scan on an extragalactic check source, J1905+0952 $\left(0.9^{\circ}\right.$ from both XTE J1908+094 and $\mathrm{J} 1907+0907)$. Observation durations ranged from $75 \mathrm{~min}$ to $4 \mathrm{hr}$, and for all observations longer than $3.5 \mathrm{hr}$, we spent 30 min at the start and end of the run observing a geodetic block, using multiple calibrator sources spread across the sky to remove residual tropospheric delays and clock errors, to improve the accuracy of our phase referencing.

The data were correlated using the VLBA-DiFX software correlator (Deller et al. 2007), and reduced using standard procedures within the Astronomical Image Processing System (AIPS; Greisen 2003).

\subsection{2 $E V N$}

In October 2013 we made three Target of Opportunity (ToO) observations using the European VLBI Network (EVN) in rapid-response e-VLBI mode (Szomoru 2008), under program codes RR007A and RR007B. As an ad hoc 'Out of Session' experiment, only six stations were available for the first observation (On, Jb, Ys, Sh, Hh \& Tr), whereas the remaining two observations were part of a regular e-VLBI session, with ten stations available (Ef, Jb, Mc, 
Table 1. Results of VLBI observations. VLBA and EVN observations begin with project codes BM and RR respectively. $S$ and $N$ denote southern and northern components, respectively, for the one epoch in which both components were detected.

\begin{tabular}{lccccccrr}
\hline $\begin{array}{l}\text { Epoch } \\
\text { (MJD) }\end{array}$ & $\begin{array}{c}\text { Start date } \\
\text { (yyyy-mm-dd) }\end{array}$ & $\begin{array}{c}\text { UT range } \\
(\text { hh:mm-hh:mm })\end{array}$ & $\begin{array}{c}\text { Project } \\
\text { code }\end{array}$ & $\begin{array}{c}\text { Freq. } \\
(\mathrm{GHz})\end{array}$ & $\begin{array}{c}\text { Beam size } \\
(\text { mas } \times \text { mas })\end{array}$ & $\begin{array}{c}\text { Peak flux } \\
\left(\mu \mathrm{Jy} \mathrm{bm}^{-1}\right)\end{array}$ & $\begin{array}{c}\text { Total flux } \\
(\mu \mathrm{Jy})\end{array}$ & $\begin{array}{c}\text { RMS noise } \\
\left(\mu \mathrm{Jy} \mathrm{bm}^{-1}\right)\end{array}$ \\
\hline 56603.78 & $2013-11-07$ & $18: 07-19: 11$ & BM382A & 8.3 & $3.23 \times 2.11$ & $607 \pm 38$ & $759 \pm 77$ & 34 \\
56604.62 & $2013-11-08$ & $11: 16-18: 43$ & RR007A & 4.9 & $8.75 \times 6.52$ & $1290 \pm 102$ & $1200 \pm 130$ & 112 \\
56604.82 & $2013-11-08$ & $18: 36-20: 53$ & BM382B & 8.3 & $2.71 \times 1.59$ & $2342 \pm 22$ & $3637 \pm 95$ & 18 \\
56605.79 & $2013-11-09$ & $18: 10-19: 39$ & BM382C & 8.3 & $3.28 \times 1.93$ & $1799 \pm 42$ & $7898 \pm 223$ & 31 \\
56606.80 & $2013-11-10$ & $17: 37-20: 39$ & BM382D & 8.3 & $3.53 \times 1.64$ & $2254 \pm 36$ & $15054 \pm 462$ & 31 \\
$56607.78^{S}$ & $2013-11-11$ & $17: 37-19: 39$ & BM382E & 8.3 & $4.01 \times 1.96$ & $386 \pm 44$ & $2829 \pm 394$ & 34 \\
$56607.78^{N}$ & & & & & & $921 \pm 34$ & $1468 \pm 82$ & 34 \\
56608.64 & $2013-11-12$ & $12: 12-18: 42$ & RR007B & 4.9 & $7.44 \times 6.27$ & $690 \pm 8$ & $940 \pm 30$ & 8 \\
56609.00 & $2013-11-13$ & $22: 06-00: 54$ & BM382F & 8.3 & $3.56 \times 1.00$ & $680 \pm 30$ & $1851 \pm 631$ & 19 \\
56609.54 & $2013-11-13$ & $10: 21-12: 58$ & RR007B & 4.9 & $12.92 \times 5.76$ & $267 \pm 9$ & $544 \pm 20$ & 9 \\
56610.90 & $2013-11-14$ & $20: 07-22: 53$ & BM382G & 8.3 & $2.43 \times 1.13$ & $<84$ & N/A & 28 \\
56612.92 & $2013-11-16$ & $20: 38-23: 24$ & BM382H & 8.3 & $2.50 \times 0.95$ & $<135$ & N/A & 45 \\
56617.90 & $2013-11-21$ & $20: 09-22: 55$ & BM382I & 8.3 & $2.29 \times 0.88$ & $<100$ & N/A & 33 \\
56742.25 & $2014-05-14$ & $02: 09-09: 41$ & RR009 & 4.9 & $5.87 \times 4.72$ & $<65$ & N/A & 22 \\
\hline
\end{tabular}

Nt, On, Tr, Ys, Wb, Sh \& Hh). As such, although the target was detected in all three observations, it was not possible to unambiguously determine the astrometric position of the first epoch (MJD 56604.6; RR007A) due to its poor $u v$-coverage. All observations were performed with a data transmission rate of $1024 \mathrm{Mbps}$ using dual polarisation, giving a total bandwidth of $128 \mathrm{MHz}$ centred at a frequency of $4.9 \mathrm{GHz}$ and correlated by the EVN Software Correlator at JIVE (Keimpema et al. 2015). The observations were phase referenced to J1905+0952, and we included brief scans on J1907+0907 as a positional check source. The cycle time was $4.5 \mathrm{~min}$, with each cycle comprising $2 \mathrm{~min}$ on the calibrator and $2.5 \mathrm{~min}$ on the target.

During the first half of 2014 the target appeared to return to the hard state and we requested an additional eVLBI ToO observation that was taken in May 2014 with the EVN (project code RR009). The EVN did not detect the source during this experiment.

All EVN data calibration was initially performed using the JIVE/EVN pipeline (Reynolds et al. 2002) which uses PARSELTONGUE (Kettenis et al. 2006), a python wrapper for the NRAO Astronomical Image Processing System (AIPS). Standard delay, phase and amplitude solutions were derived from bright standard calibrator sources and interpolated to the two nearby reference sources. Further time-dependent phase solutions were calculated for J1905+0952, which in turn were interpolated to J1907+0907 and the target, for astrometric reference. The four epochs of the target field were edited for bad data points and imaged using a Briggs weighting of 1 . As with our VLBA data, an image plane deconvolution of the synthesised beam was performed for each epoch to fit the centroid position and measure any extension of the radio emission.

\subsection{Astrometric correction}

While all astrometric positions were measured relative to a phase calibrator, the VLBA and EVN used different phase reference sources (J1907+0907 and J1905+0952, respectively). They were also observed at different frequencies, so any core shift in the calibrator due to a resolved jet would result in an astrometric shift in the target image.
We therefore compared the relative positions of the two calibrator sources in each data set, which was possible since in each case the phase reference calibrator for one array was used as the check source for the other. In Figure 1 we show the relative positions of each calibrator as measured by the EVN (in red) and VLBA (in blue). We determined a mean offset of the EVN frame relative to that of the VLBA of $-2.14 \pm 0.14$ mas in R.A. and $1.87 \pm 0.13$ mas in Dec., and a mean offset of the VLBA frame relative to that of the EVN of $1.73 \pm 0.08$ mas in R.A. and $-1.56 \pm 0.13$ mas in Dec..

The nominal uncertainties on the known calibrator positions $^{1}$ are $<0.3$ mas, so cannot alone explain the measured offsets. However, J1905+0952 was resolved by the EVN (Figure 2), suggesting that the core location measured at $5 \mathrm{GHz}$ by the EVN could be shifted downstream along the jet axis as compared to the $8.4-\mathrm{GHz}$ position measured by the VLBA. The poorly-modelled tropospheric delay in the EVN observations could have introduced a positional shift in declination, and the combination of these two effects, in addition to the high winds prevailing at many of the EVN stations during the observations, could potentially explain the observed shift between the EVN and VLBA frames.

To enable a valid comparison of the positions measured by the two arrays, we therefore attempted to align the two sets of observations onto the same astrometric frame. Since the offsets measured independently by each telescope overlapped within the uncertainties, then through the rest of this paper we use the average offsets of $\Delta \alpha=1.9$ mas and $\Delta \delta=-1.7$ mas to correct the measured EVN positions to the VLBA reference frame.

\section{RESULTS AND ANALYSIS}

The first VLBI observations were taken approximately eight days after the first radio detection of the 2013 outburst (the images are shown in Figure 3). The high-resolution observations initially detected a relatively compact, slightly resolved radio component, with a fitted angular size of $2.2 \pm 0.8$ mas.

1 http://astrogeo.org/ 


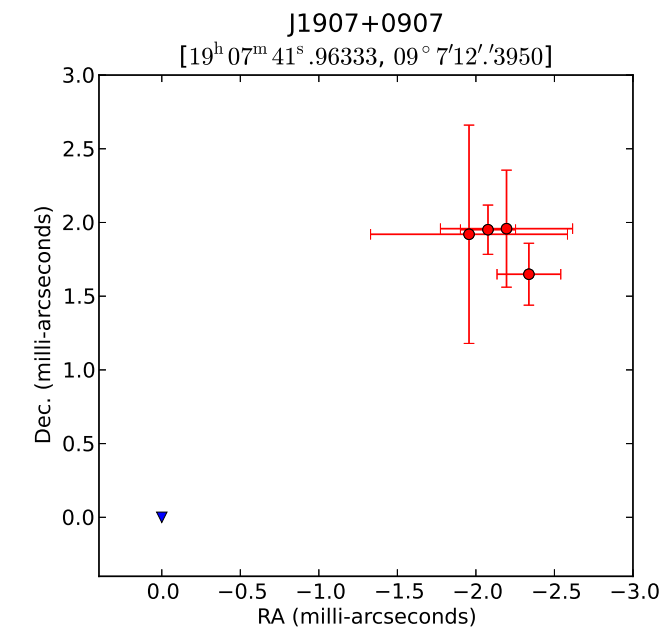

$1905+0952$

$\left[19^{\mathrm{h}} 05^{\mathrm{m}} 39^{\mathrm{s}} .89887,09^{\circ} 52^{\prime} 08^{\prime \prime} .4077\right]$

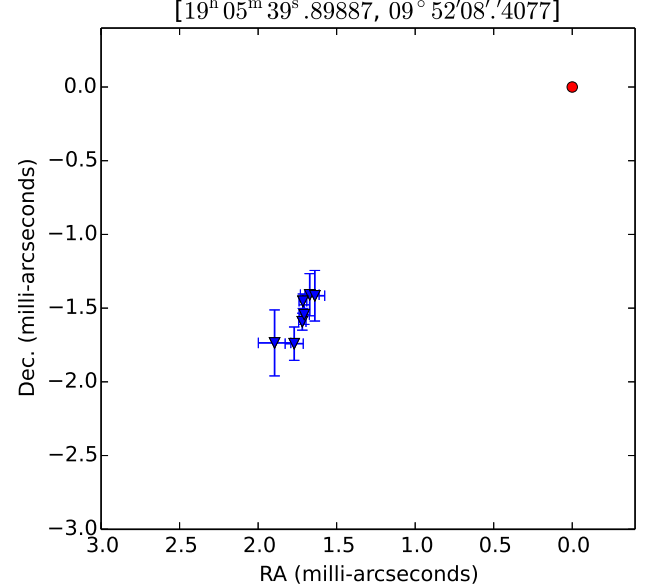

Figure 1. The measured offsets of the check sources from their known positions, as measured by the EVN and VLBA. The top panel shows the difference between the position used at correlation for J1907+0907 (blue), and the observed position (red) when phase-referenced to J1905+0952 in EVN experiments. The bottom panel shows the difference between the position used at correlation for J1905+0952 (red) and the observed position (blue) when phase referenced to J1907+0907 in VLBA experiments. We use the mean offsets to correct the EVN measured positions to the VLBA frame.

Over the course of the next four days the component expanded and moved towards the south. As it did so, the component developed a more complex substructure. Rather than a single, resolved Gaussian, a "core-halo" morphology was detected on MJD 56606.8, with a broad, diffuse structure surrounding an inner hot-spot. Four days after the initial detection, the southern component had expanded so much that its surface brightness began to fade below detectability.

As the southern component expanded and became resolved out, a new component appeared $\sim 20$ mas to the north. This northern component moved in the opposite direction, and also started to expand. However, it did not appear to show quite the same level of complexity as the southern component; although two of the VLBI observations were taken at a lower resolution with the EVN (due to the lower observing frequency and shorter baselines), the two VLBA epochs showed a much more compact morphology, without

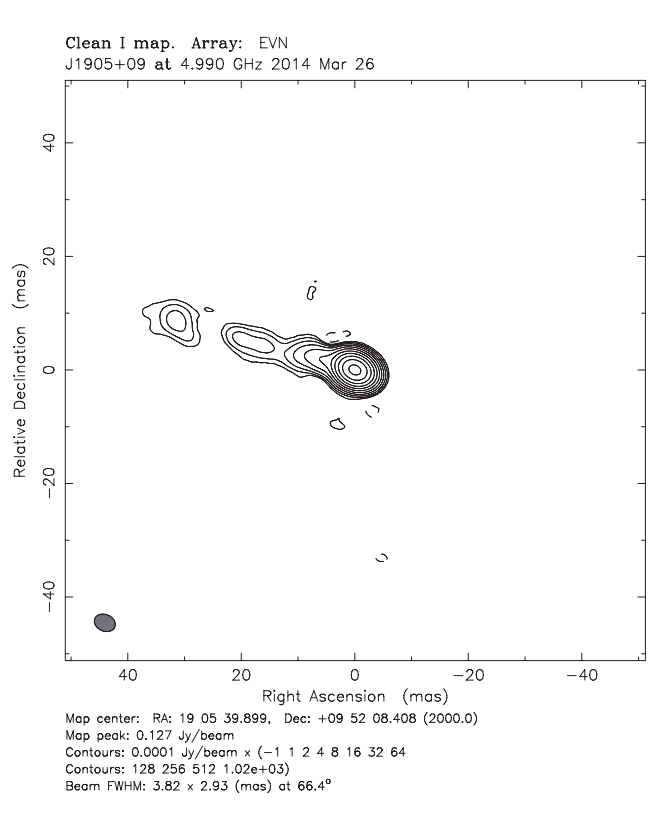

Figure 2. $5 \mathrm{GHz}$ EVN image showing the resolved jet of the calibrator source $\mathrm{J} 1905+0952$. The peak brightness is $127 \mathrm{mJy} \mathrm{bm}^{-1}$, and contours are at levels of $-1,1,2,4,8,16,32, \ldots, 512$ times the lowest contour of $0.1 \mathrm{mJy} \mathrm{bm}^{-1}$. The offsets measured in Fig. 1 are not aligned with the jet axis, but could potentially be explained by a combination of the resolved calibrator and unmodelled tropospheric delays that become significant at low elevations.

the development of a prominent core-halo structure as seen in the southern component. Furthermore, both the peak and integrated flux of this northern component remained much lower than those of its southern counterpart.

The rise and fall of the large radio flare reported by Curran et al. (2015) are contemporaneous with our VLBI observations, and can therefore be explained not as a single event, but as the brightening and fading of two distinct, resolved ejecta. The two swings in the electric vector position angle (EVPA) of the linear polarization detected by Curran et al. (2015) could also be related to the appearance and evolution of the different components. This will be explored in more detail in Section 4.1.2.

\subsection{Motion of resolved components}

Our VLBI observations were triggered too late to detect the "steady" jet emission from the core and we can place an upper limit of $\lesssim 30 \mu J y$ on the core brightness in our deepest epoch on MJD 56608-9 (EVN observation RR007B). While this precludes an unambiguous determination of the location of the central binary system, we are nonetheless able to estimate the core position by extrapolating the motions of the ballistically-moving ejecta backwards in time until they intersect. To do so, we used the measured positions of the peak flux density in each image to estimate the precise position of the binary at each epoch (Table 2), and then independently fit the positions of the northern and southern components with a linear motion (Figure 4). While it is possible that the northern and southern components were initially ejected at 


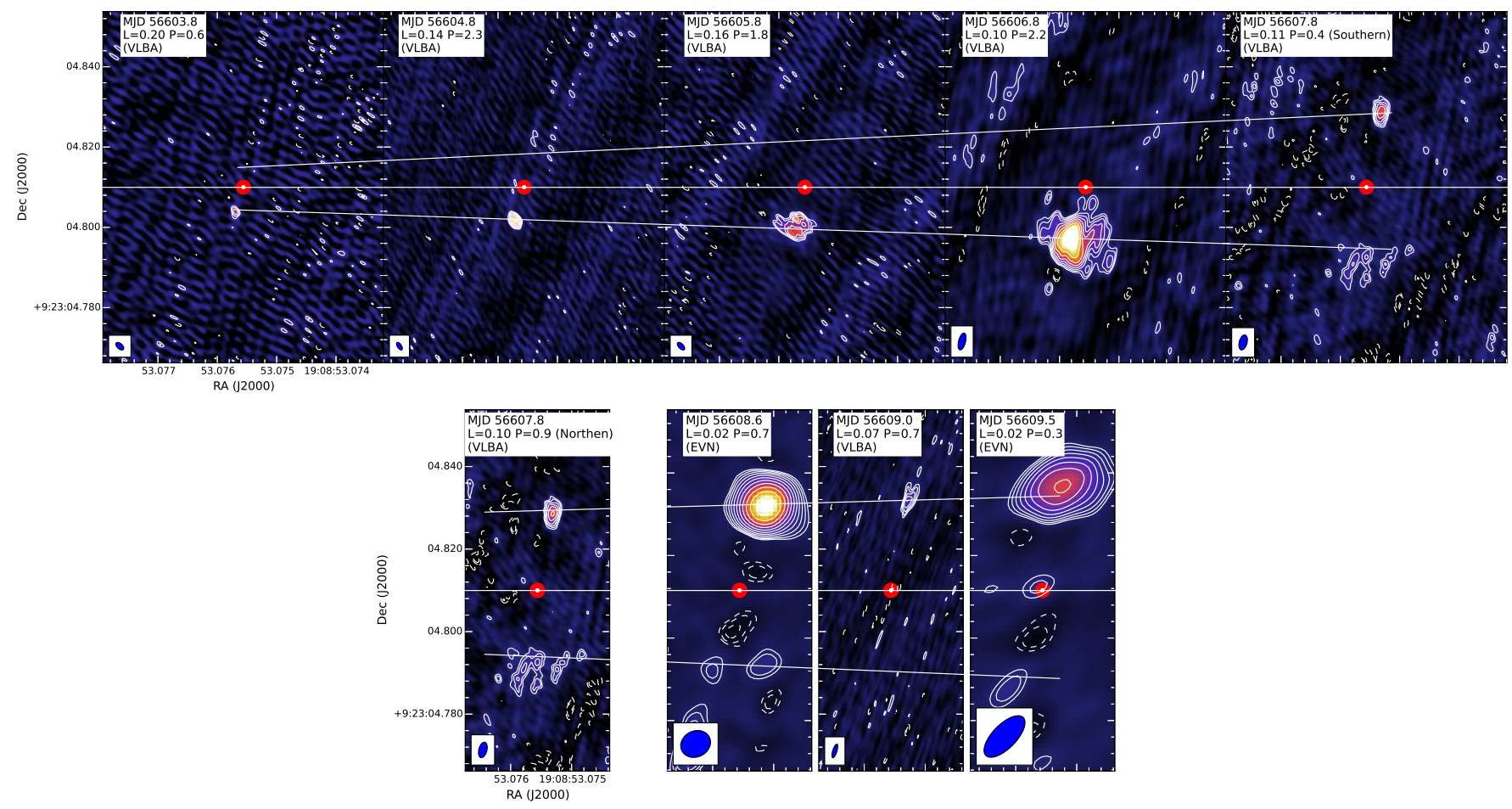

Figure 3. VLBI maps of XTE J1908+094 during the 2013 outburst, all taken following the hard-to-soft X-ray state transition. The top panels highlight the evolution of the southern component and span 4 days in time, up to its disappearance following the VLBA epoch on MJD 56607.8. The bottom panels highlight the evolution of the northern component from its first appearance on the same day (MJD 56607.8), and span 1.7 days in time. $\mathrm{L}$ and $\mathrm{P}$ indicate the lowest and peak contours in units of mJy bm ${ }^{-1}$, with contour levels of $\pm(\sqrt{2})^{n}$, where $n=3,4,5,6, \ldots$. The red circle indicates the estimated position of the binary (Section 3.1) and the white lines show the fitted motions of the respective components. We see clear expansion of the southern component over time, and a delay in the appearance of the northern component.

Table 2. Astrometric results

\begin{tabular}{|c|c|c|}
\hline \multirow{2}{*}{$\begin{array}{l}\text { Epoch } \\
\text { (MJD) }\end{array}$} & \multicolumn{2}{|c|}{ Peak position } \\
\hline & RA (19h 08m ss.sssssss) & Dec $\left(09^{\circ} 23^{\prime}\right.$ ss.ssssss $)$ \\
\hline 56603.78 & $53.0756925 \pm 0.00018 s$ & $04.804002 \pm 0.0027^{\prime \prime}$ \\
\hline 56604.62 & $\mathrm{~N} / \mathrm{A}$ & $\mathrm{N} / \mathrm{A}$ \\
\hline 56604.82 & $53.0757174 \pm 0.00014 s$ & $04.801382 \pm 0.0022 "$ \\
\hline 56605.79 & $53.0757372 \pm 0.00017 \mathrm{~s}$ & $04.799978 \pm 0.0026 "$ \\
\hline 56606.80 & $53.0757841 \pm 0.00017 \mathrm{~s}$ & $04.797786 \pm 0.0026 "$ \\
\hline $56607.78^{S}$ & $53.0757905 \pm 0.00020 \mathrm{~s}$ & $04.791226 \pm 0.0030 "$ \\
\hline $56607.78^{N}$ & $53.0753187 \pm 0.00020 \mathrm{~s}$ & $04.828601 \pm 0.0030 "$ \\
\hline 56608.64 & $53.0751346 \pm 0.00020 \mathrm{~s}$ & $04.832090 \pm 0.0030 "$ \\
\hline 56609.00 & $53.0752967 \pm 0.00015 s$ & $04.831471 \pm 0.0023 "$ \\
\hline 56609.54 & $53.0752819 \pm 0.00062 \mathrm{~s}$ & $04.836453 \pm 0.0093 "$ \\
\hline 56610.90 & $\mathrm{~N} / \mathrm{A}$ & $\mathrm{N} / \mathrm{A}$ \\
\hline 56612.92 & $\mathrm{~N} / \mathrm{A}$ & $\mathrm{N} / \mathrm{A}$ \\
\hline 56617.90 & $\mathrm{~N} / \mathrm{A}$ & $\mathrm{N} / \mathrm{A}$ \\
\hline 56742.25 & $\mathrm{~N} / \mathrm{A}$ & $\mathrm{N} / \mathrm{A}$ \\
\hline
\end{tabular}

different epochs, this seems unlikely based on our knowledge of typical X-ray binary jet properties. It is also unlikely that the components were ejected before the X-ray state change, which began on or prior to MJD 56597.92 (Zhang et al. 2015).

The fitted proper motion of the southern component was $\mu_{\mathrm{S}}=1.88 \pm 0.08$ mas day $^{-1}$ at a position angle of $167.5 \pm 0.6^{\circ}$ east of north. Our fit to the motion of the northern component gave $\mu_{\mathrm{N}}=2.40 \pm 0.35$ mas day $^{-1}$ along a position angle of $-11.1 \pm 2.8^{\circ}$, consistent with it being the bipolar counterpart of the southern component. This led to an inferred position for the central binary of (J2000) RA $19^{\mathrm{h}} 08^{\mathrm{m}} 53^{\mathrm{s}} .07556 \pm 0.00004$, Dec. $+9^{\circ} 23^{\prime} 04^{\prime \prime} 810 \pm 0^{\prime \prime} 002$ (marked as a red circle in Figure 3) and an ejection date of $t_{0}=$ MJD 56600.0 \pm 0.7 . We note that the southern component first appears significantly closer to the estimated binary position than the first detection of its northern counterpart.

Assuming an intrinsically symmetric ejection event we can use the approaching and receding proper motions $\left(\mu_{\mathrm{a}}\right.$ and $\mu_{\mathrm{r}}$, respectively) to constrain the intrinsic jet velocity 


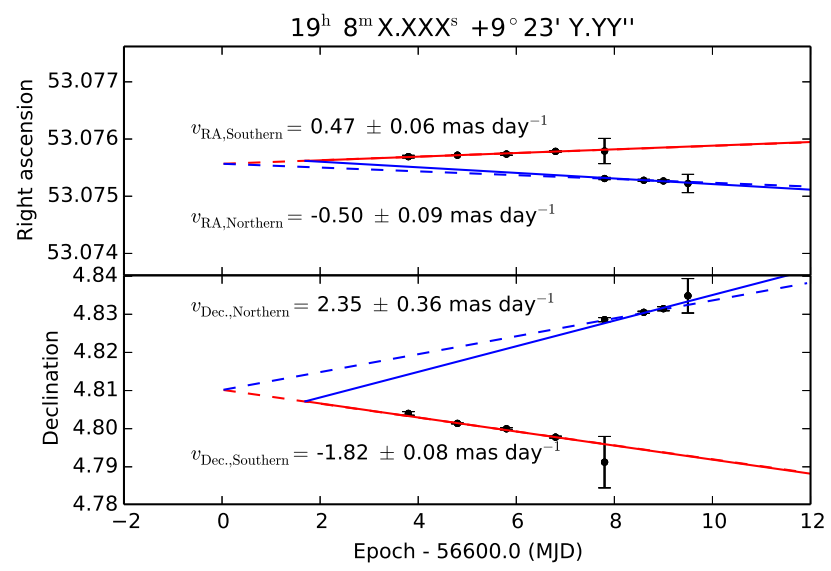

Figure 4. Astrometric positions of the components. The red and blue lines are weighted best fits to the southern and northern components respectively, assuming they are ejected simultaneously and exhibit ballistic motion. The dashed lines indicate the best fit with $t_{0}$ as a free parameter (fitted as MJD 56600.0), and correspond to the proper motions stated on the plot. The solid lines used a fixed ejection time (corresponding to the prompt radio flare at MJD 56601.7), and give a slightly higher velocity in declination for the northern component of $v_{\text {Northern }}=3.35 \pm 0.45$ mas day $^{-1}$. Both components appear to exhibit ballistic motion.

and inclination angle via

$\mu_{\mathrm{r}, \mathrm{a}}=\frac{\beta \sin \theta}{1 \pm \beta \cos \theta}$,

which leads to the constraint

$\beta \cos \theta=\frac{\mu_{\mathrm{a}}-\mu_{\mathrm{r}}}{\mu_{\mathrm{a}}+\mu_{\mathrm{r}}}$

where $\beta=v / c$ is the jet speed as a fraction of the speed of light and $\theta$ is the inclination angle of the jet axis to the line of sight. From the measured proper motions and assuming that the faster-moving northern component is the approaching one, this would imply $\beta \cos \theta=0.12 \pm 0.07$. Therefore, since both $\beta<1$ and $\cos \theta<1$ we constrain the intrinsic velocity to be $<0.19 c$ and the inclination angle $\theta>79^{\circ}$, (both at $1 \sigma$ confidence). Moreover, at a distance of $8 \mathrm{kpc}$, the projected separation speed of the components in the plane of the sky is $<0.2 c$, reinforcing the conclusion of a low jet velocity if the components are intrinsically symmetric ejecta modified by relativistic boosting and light travel time effects.

Given that our derived ejection date was several days before any significant changes in the overall radio light curve, we also re-fit the component motions assuming the ejection to have taken place at the time of the rapid radio flare seen by AMI-LA on MJD 56601.7 (Curran et al. 2015). Given the high-cadence VLBA sampling of the southern component, this assumption only significantly changed the velocity of the northern knot, which increased to 3.4 mas day $^{-1}$ (see Figure 4). Such a high proper motion relative to the southern component would suggest that if the ejecta did correspond to the initial radio flare at MJD 56601.7, then the northern component is likely to be the approaching one.
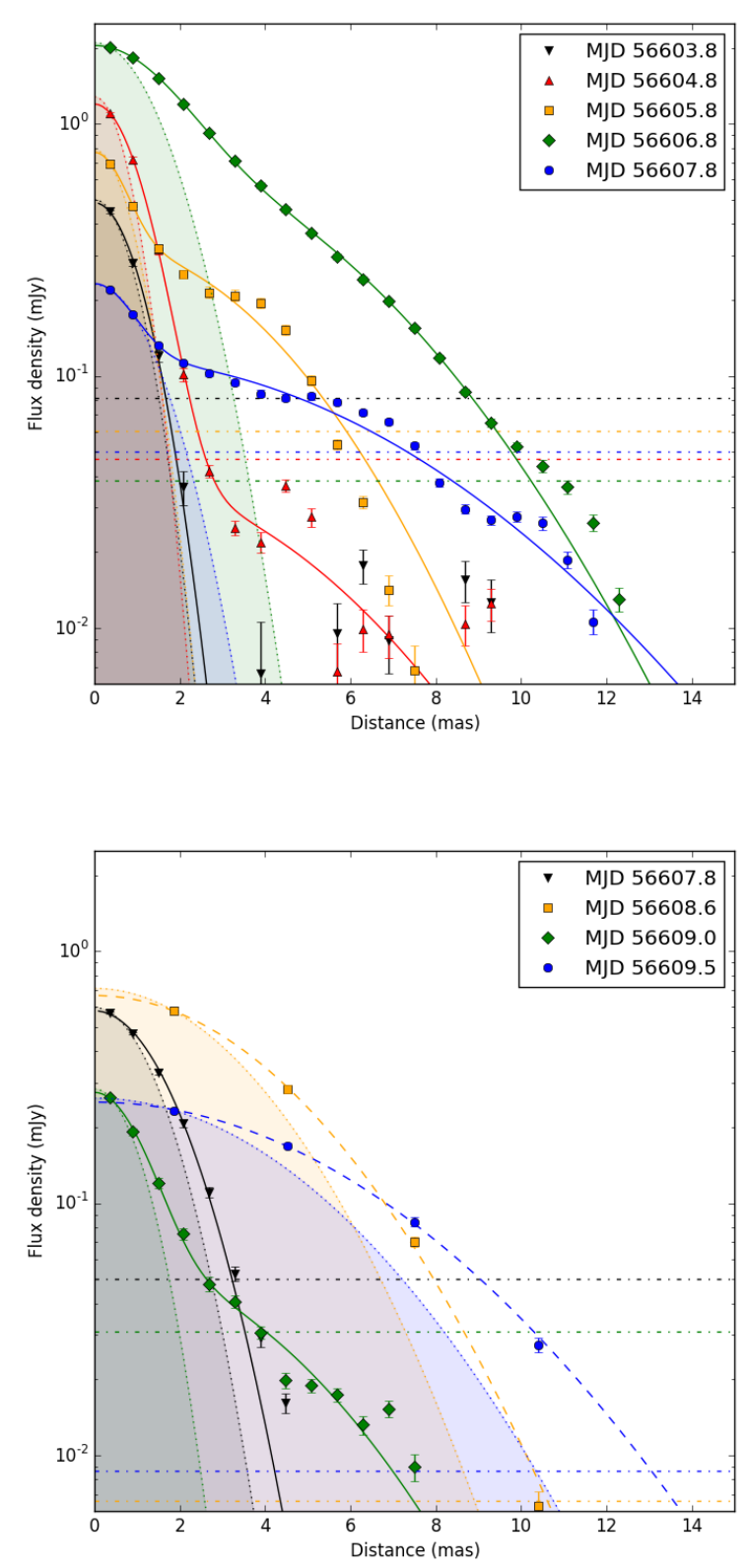

Figure 5. Radial profiles of the extended southern (top panel) and northern (bottom panel) ejecta, derived using the AIPS task IRING. We fit each profile with one-dimensional Gaussian models centred on the position of peak flux in each image. The solid lines are fits to the $8.4-\mathrm{GHz}$ VLBA data and the dashed lines are fits to the $5-\mathrm{GHz}$ EVN data. The horizontal dot-dashed lines show the RMS noise level of each epoch. The shaded regions represent the minimum beam size in each image, as a guide to the intrinsic angular resolution of the images. In several of the epochs we see clear evidence for two components, corresponding to a core-halo morphology. 


\subsection{Expansion of resolved components}

As discussed in Section 3, the two components expanded as they moved outwards. Figure 3 shows that the southern knot was initially very compact. It then expanded as it moved away from the central binary system, developing a bright core and extended halo before becoming resolved out by MJD 56608. The initial VLBA detection of the northern component was slightly resolved, and by the time of the second observation it had expanded to $8.3 \times 1.1 \mathrm{mas}^{2}$ in size (Table 3). Unfortunately, the lower resolution of the EVN makes it difficult to further quantify the expansion of the northern component.

The emitting regions became resolved over the course of the observing campaign, and were not always well approximated by standard elliptical Gaussian models, making it difficult to quantify the source size and hence expansion rate. To get an initial idea of the extension of the components at each epoch, we first used the AIPS task IRING. This task integrates the flux contained within successive concentric annuli, which we centred on the position of peak flux density in a given image. This provided an azimuthally-averaged estimate of the radial extent of each component, providing a first-order size estimate, which was particularly useful for the most heavily-resolved epochs. We chose the width of each annulus to be 3 pixels (equivalent to $0.6-0.9$ mas for the VLBA and 3 mas for the EVN), and the results are shown in Figure 5. In an attempt to parametrize the resulting radial profiles, we tried fitting them with a variety of simple onedimensional models (Gaussian, Lorentzian and power law functions) and examined the residuals. We found the best fits when using either a one- or two-component Gaussian function centred on the peak emission, which gave residuals consistent with the image noise.

The two-Gaussian fits correspond to a 'core-halo' morphology, suggesting that while some of the flux remained unresolved it was surrounded by more diffuse emission. This 'halo' seemed to extend up to $\sim 20$ mas in diameter and was not present in the first epoch. More detailed inspection of the individual images (Fig. 3) showed the halo to be relatively isotropic (i.e. not significantly elongated parallel or perpendicular to the position angle of the jet) and to move with the bulk motion of the ejecta.

Using the AIPS task JMFIT we fitted the images with either one or two elliptical Gaussians depending on the best IRING fit (as listed in table 3). The results of this twodimensional image-plane analysis showed a slight tendency for the major axis of the elliptical Gaussians to be aligned along a position angle close to the jet direction of $\sim 167^{\circ} \mathrm{E}$ of $\mathrm{N}$. When fitting the mean sizes of each component over time, the outer halo structure of the southern component was found to expand linearly at a rate of $3.2 \pm 0.5$ mas day $^{-1}$ (Fig. 6). Midway through the expansion, the core component rebrightened and became marginally resolved, disappearing again $\sim 36 \mathrm{hrs}$ later. For the northern component we only fit an expansion rate to the $5-\mathrm{GHz}$ observations, as it is not meaningful to compare sizes at different wavelengths. This gave an expansion rate of $2.9 \pm 0.1$ mas day $^{-1}$. Thus, the northern and southern components appeared to have similar expansion rates of $\sim 3$ mas day ${ }^{-1}$, corresponding to roughly $0.15 c$ at $8 \mathrm{kpc}$.

In the VLBA epoch on MJD 56609.0, we also attempted

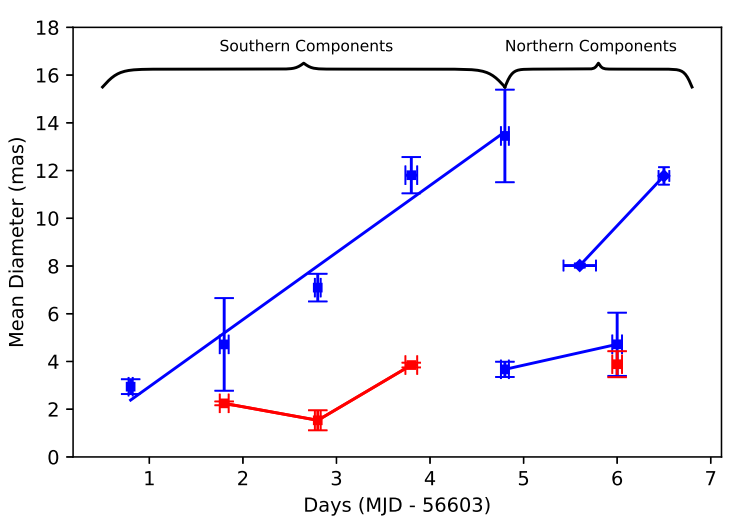

Figure 6. Expansion of the two ejected components. The blue points are the mean diameter of the surrounding 'halo' structure and the red points are the epochs that also displayed an inner 'core' component. The southern halo appears to expand at a constant rate.

a two-Gaussian fit to the northern component, guided by the IRING results (see Fig. 5), which tentatively suggested a core-halo structure. To test the reality of this hypothesis, we also conducted model fitting of the visibility data within the DIFMAP software package (Shepherd 1997). While DIFMAP does not return a formal uncertainty on the derived parameters, by model-fitting in the visibility domain it can rule out any spurious structures arising from imaging artefacts. In this case the Difmap fits did warrant a two-Gaussian fit, giving us more confidence in the development of a similar diffuse "halo" structure around the northern component.

\section{DISCUSSION}

Our VLBI observations were made following the hard-tosoft X-ray spectral state transition at the peak of the 2013 outburst of XTE J1908+094. We detected and tracked a set of ballistically-moving ejecta that expanded on moving away from the core, and directly resolved the jets perpendicular to the direction of motion. Although our VLBI observations did not detect the "steady" jet emission from the core that is typically seen in the hard/plateau state (e.g. GRS 1915+105; Dhawan et al. 2000; Rushton et al. 2010), we were able to infer its location by extrapolating the proper motions of the ballistically-moving components back to zero separation.

To explain the observed properties of the evolving ejecta, we now briefly review our main findings in the context of the standard models for X-ray binary jets. We then combine our imaging results with the radio light curves, spectra and polarimetry presented by Curran et al. (2015), to arrive at a preferred scenario for the observed evolution of the jets in XTE J1908+094.

\subsection{Key observational signatures}

\subsubsection{North/south asymmetry}

There are clear differences in the properties and evolution of the northern and southern components. The southern com- 
Table 3. JMFIT results showing the fitted sizes of the Southern \& Northern components. Each component was fitted with either one or two Gaussians, as indicated. Two-Gaussian fits correspond to the epochs with a core-halo morphology. $\theta_{\text {maj }}, \theta_{\text {min }}$ and $P A$ are respectively the major and minor axes and position angle of the fitted elliptical Gaussians.

\begin{tabular}{|c|c|c|c|c|c|c|c|c|c|}
\hline & $\begin{array}{l}\text { Epoch } \\
\text { (MJD) }\end{array}$ & $\begin{array}{l}\theta_{\mathrm{maj}, 1} \\
\text { (mas) }\end{array}$ & $\begin{array}{l}\theta_{\min , 1} \\
(\mathrm{mas})\end{array}$ & $\begin{array}{l}P A_{1} \\
\text { (deg.) }\end{array}$ & $\begin{array}{l}\text { Total flux } 1 \\
\text { (mJy) }\end{array}$ & $\begin{array}{l}\theta_{\mathrm{maj}, 2} \\
\text { (mas) }\end{array}$ & $\begin{array}{l}\theta_{\min , 2} \\
(\mathrm{mas})\end{array}$ & $\begin{array}{l}P A_{2} \\
\text { (deg.) }\end{array}$ & $\begin{array}{c}\text { Total flux } 2 \\
\text { (mJy) }\end{array}$ \\
\hline \multirow{5}{*}{ 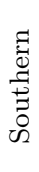 } & $56603.78 \pm 0.02$ & $3.79 \pm 0.40$ & $2.10 \pm 0.22$ & $158 \pm 7$ & $0.74 \pm 0.13$ & & & & \\
\hline & $56604.82 \pm 0.05$ & $6.91 \pm 2.84$ & $2.52 \pm 1.04$ & $163 \pm 14$ & $0.89 \pm 0.41$ & $2.55 \pm 0.09$ & $1.94 \pm 0.07$ & $30 \pm$ & $3.10 \pm 0.15$ \\
\hline & $56605.79 \pm 0.03$ & $7.98 \pm 0.65$ & $6.21 \pm 0.51$ & $161 \pm 13$ & $9.08 \pm 0.78$ & $2.05 \pm 0.56$ & $1.02 \pm 0.28$ & $46 \pm 15$ & $<0.27$ \\
\hline & $56606.80 \pm 0.06$ & $14.24 \pm 0.92$ & $9.37 \pm 0.60$ & $103 \pm 6$ & $10.94 \pm 0.74$ & $4.22 \pm 0.11$ & $3.48 \pm 0.09$ & $160 \pm 5$ & $4.69 \pm 0.16$ \\
\hline & $56607.78 \pm 0.04$ & $15.11 \pm 2.18$ & $11.79 \pm 1.70$ & $160 \pm 5$ & $4.72 \pm 0.71$ & & & & \\
\hline \multirow{4}{*}{ 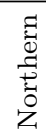 } & $56607.78 \pm 0.04$ & $4.69 \pm 0.41$ & $2.65 \pm 0.23$ & $175 \pm 6$ & $0.97 \pm 0.13$ & & & & \\
\hline & $56608.64 \pm 0.17$ & $8.32 \pm 0.07$ & $7.72 \pm 0.10$ & $112 \pm 5$ & $0.94 \pm 0.13$ & & & & \\
\hline & $56609.00 \pm 0.05$ & $8.30 \pm 2.33$ & $1.14 \pm 0.32$ & $149 \pm 4$ & $0.30 \pm 0.11$ & $5.83 \pm 0.82$ & $1.94 \pm 0.27$ & $174 \pm 3$ & $0.70 \pm 0.13$ \\
\hline & $56609.54 \pm 0.06$ & $14.22 \pm 0.44$ & $9.33 \pm 0.29$ & $14 \pm 3$ & $0.48 \pm 0.02$ & & & & \\
\hline
\end{tabular}

ponent appears four days earlier than its northern counterpart, and at its maximum flux density is also significantly brighter than the peak emission from the northern component. Furthermore, the southern component persists for longer, and appears to be more extended.

Differences in the flux densities of approaching and receding ejecta in X-ray binary jets are often ascribed to Doppler boosting, such that the approaching component appears significantly brighter than the receding component. This explanation would lead us to infer that the southern component was approaching and the northern component receding. While this would initially seem to explain both the observed differences in peak flux density and the delayed appearance of the northern component (due to light travel time effects), such an explanation would be at odds with the lower proper motion measured for the southern component (Section 3.1).

Assuming that the two components were intrinsically identical, were ejected at the same time, and moved ballistically, the observed proper motions of both components then constrain $\beta \cos \theta=0.12 \pm 0.07$. This implies a low jet speed, a motion close to the plane of the sky, or both. We note that the X-ray spectral fitting of Miller et al. (2009), Tao et al. (2015), and Zhang et al. (2015) determined an inclination angle of the inner disc (presumably aligned with the black hole spin and hence with the jet axis) that lies in the range $20-53^{\circ}$, making it unlikely that the jets are close to the plane of the sky. At a fiducial distance of $8 \mathrm{kpc}$, a proper motion of $1 \mathrm{mas} \mathrm{d}^{-1}$ would imply a projected velocity of $0.046 \mathrm{c}$ in the plane of the sky. Thus, unless XTE J1908+094 is significantly more distant than $8 \mathrm{kpc}$, the measured proper motions of $\mu_{\mathrm{N}}=2.40 \pm 0.35 \mathrm{mas} \mathrm{d}^{-1}$ and $\mu_{\mathrm{S}}=1.88 \pm 0.08 \mathrm{mas} \mathrm{d}^{-1}$ indeed imply a jet speed significantly lower than observed in other BH XRBs such as GRS 1915+105 (Mirabel \& Rodríguez 1994), GRO J1655-40 (Hjellming \& Rupen 1995), or H1743-322 (Miller-Jones et al. 2012).

Furthermore, under the above assumptions and using the measured constraint on $\beta \cos \theta$, we can determine the expected flux density ratio between the northern and southern ejecta. At equal angular separations (e.g. Miller-Jones, Blundell, \& Duffy 2004b), the ratio of flux densities should be given by

$\frac{S_{\mathrm{app}}}{S_{\mathrm{rec}}}=\left(\frac{1+\beta \cos \theta}{1-\beta \cos \theta}\right)^{k-\alpha}$,

where $k=3$ for discrete ejecta and $k=2$ for a continu- ous, steady jet, and $\alpha$ is the radio spectral index. Assuming discrete components and an appropriate spectral index of -0.6 , we would then expect a flux density ratio between the northern and southern components of 1.3-4.0. Instead, the slightly faster-moving northern component (see Fig. 4) has a flux density that is significantly lower than that of the southern component. Specifically, on MJD 56607.8, when both components have the same angular separation from the core within uncertainties, the integrated flux from the southern component is a factor $1.9 \pm 0.3$ greater than that of the northern component. In summary, the standard special relativistic effects applied to explain the behaviour of the jets in sources such as GRS 1915+105 do not appear to be solely responsible for the observed behaviour of XTE J1908+094.

The differences in the observed expansion of the two components also argue that we are not simply seeing two intrinsically symmetric jets whose observed evolution is dictated by standard relativistic boosting and light travel time delays. While our VLBA imaging shows that the southern component expands to a size of 13 mas by MJD 56607.8 (after which time it becomes resolved out), the northern component reaches a size of only 6 mas before it fades below detectability. The EVN observations, taken at a lower frequency and with shorter baselines, and hence sampling larger spatial scales, do show the northern knot to be slightly more extended. However, in the absence of similar EVN data on the southern component, we have no valid point of comparison at $5 \mathrm{GHz}$.

\subsubsection{Comparison to photometry and polarimetry}

While our VLBI imaging data provide important information as to the behaviour of the jets during the outburst, we can obtain further context from the photometric, spectral and polarimetric observations of Curran et al. (2015). The key points in the source evolution are shown in Fig. 7. The outburst began with fairly steady radio emission at around 1-2 $\mathrm{mJy}_{\text {beam }}{ }^{-1}$. This persisted until MJD 56601, when AMI-LA detected a short-lived 15 -mJy flare. The radio emission subsequently decreased to $0.3 \mathrm{mJy} \mathrm{beam}^{-1}$, before rising again from MJD 56603 to 56607. It then decayed away over the course of the next week, until the source was no longer detected.

The rise in flux density from MJD 56603-56607 coincides with the VLBI detections of the southern component, 


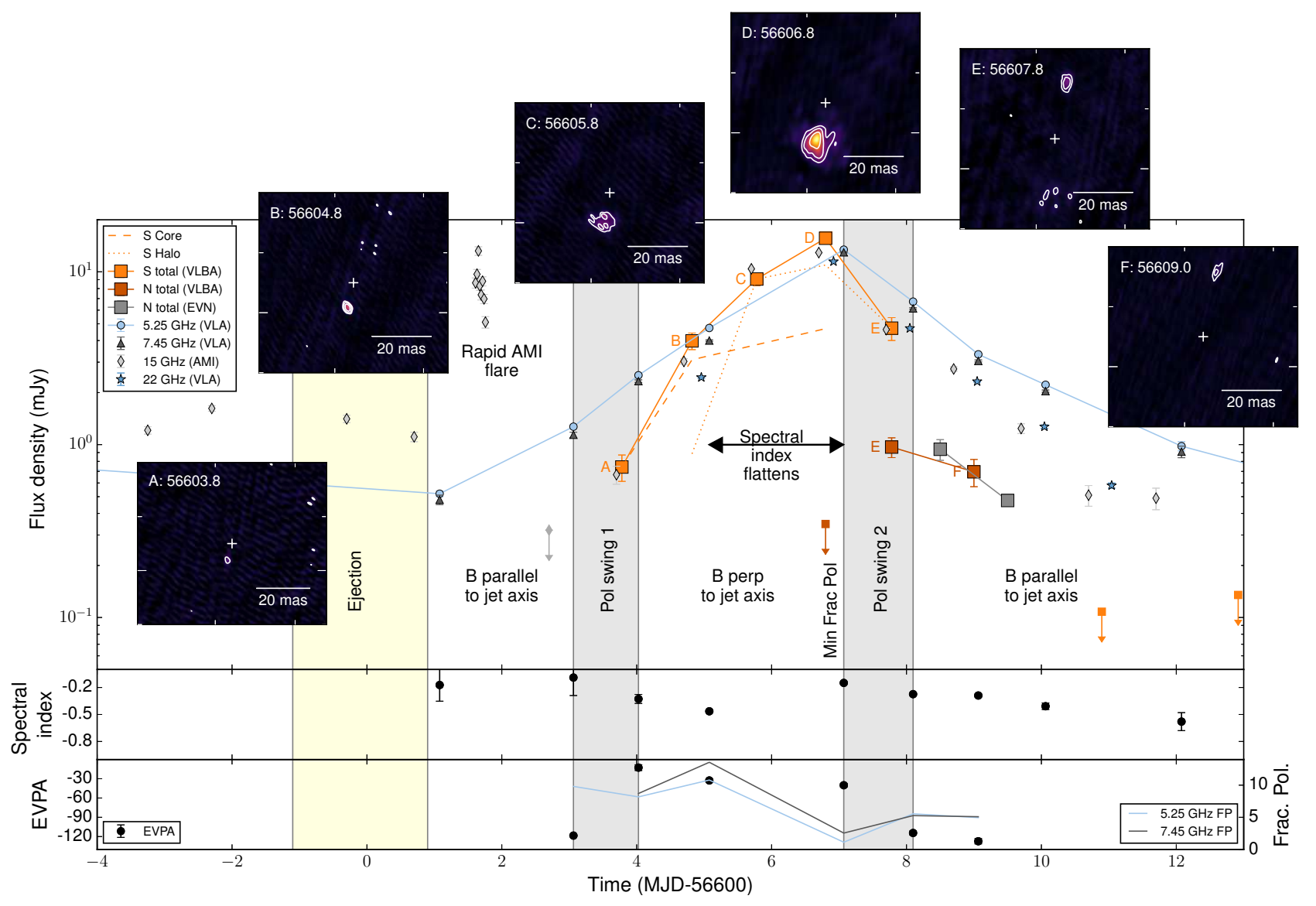

Figure 7. The evolution of the 2013 outburst of XTE J1908+094. Main panel shows the overall radio light curves, with the total flux measured by the VLBA (orange, red squares for $\mathrm{S}$ and $\mathrm{N}$ components), EVN (grey squares), VLA $(5.25 \mathrm{GHz}, 7.45 \mathrm{GHz}$ and $22 \mathrm{GHz}$ represented by blue circles, grey triangles and blue stars, respectively) and AMI (15 GHz; grey diamonds). The range of likely ejection times is shaded yellow, and the periods where $90^{\circ}$ swings of the EVPA were observed are shaded grey. Insets show the VLBA images over the six epochs (A-F) where components were detected, each of which is associated with one of the indicated light curve points on the main panel. Contours are at levels of $2^{n}$ times the rms noise, where $n=3,4,5, \ldots$ Middle panel shows the evolution of the radio spectral index and lower panel shows the evolution of the intrinsic EVPA (left axis) and the fractional polarisation (right axis), all taken from Curran et al. (2015). The large radio flare from MJD 56602-56610 is accompanied by strong evolution of the VLBI-detected components. The polarization angle swings correspond to the appearance and disappearance of the southern component, and the flattening spectral index corresponds to the re-energisation of the core of the southern component.

which gets brighter and expands over time, reaching its peak integrated flux on MJD 56606.8, before it fades and becomes resolved out (Fig. 3). Prior to the peak of the flare, our VLBI images recover virtually all of the radio emission detected by AMI and the VLA. Our first VLBA epoch recovers all of the AMI flux, although misses a few tens of percent of the flux seen several hours before and after by the VLA. During the decay phase of the flare, following the peak on MJD 56606.8, the southern component is resolved out by the VLBA, although from the light curves in Fig 7, we infer that it continues to dominate the integrated radio emission.

During the rise and decay of the main flare, Curran et al. (2015) detected significant swings in the intrinsic electric vector position angle (EVPA) of the linear polarization. This first changed during the rise phase, from $-118^{\circ}$ on MJD 56603.1 to $-13 \pm 5^{\circ}$ on MJD 56604.0. Over the next few days, as the southern component evolved and expanded, the intrinsic EVPA gradually evolved to $-40 \pm 3^{\circ}$ by MJD 56607.1, after which it showed a second large swing, to $-114 \pm 3^{\circ}$ on MJD 56608.1. This coincides with the disappearance of the southern component and the appearance of the northern component. For an optically thin synchrotron source, the EVPA should be perpendicular to the projection of the magnetic field on the plane of the sky. Since the EVPA was approximately aligned with the observed jet axis (see Section 3.1) on MJD 56604.0, we infer that the magnetic field was perpendicular to the jet axis. This would be consistent with shock compression, either from internal shocks within the jet, or from external shocks where the jets ran into the surrounding ISM. The subsequent slow rotation of the EVPA up to MJD 56607.1 corresponds to the expansion of the southern component and the decrease in the fractional polarization. This suggests that the magnetic fields in the southern knot were becoming less well aligned as the knot expanded. The second large EVPA swing corresponding to the appearance of the northern component suggests that the magnetic field in the northern component was aligned relatively well with the jet axis. This cannot be explained as 
shock compression perpendicular to the jet axis, and instead suggests a more complicated magnetic field geometry in the northern component (see, e.g., Curran et al. 2014, 2015, and discussions therein).

In addition to the photometric and polarimetric results, Curran et al. (2015) also detected a steepening spectral index between MJD 56603.1 and 56605.1 as the southern component expanded, but then saw the spectral index flatten again from -0.5 to -0.15 between MJD 56605.1 and 56607.1 , after which it steepened continuously to $\alpha=-0.8$ by MJD 56616.0. This flattening of the spectral index coincides with the development of the bright hotspot in the southern component on MJD 56606.8 (the "core" in the corehalo structure). This suggests ongoing particle acceleration at the hotspot before the emitting region expands adiabatically and the synchrotron self-absorption turnover moves to lower frequencies, giving rise to the subsequent spectral steepening. No similar flattening is seen following the appearance of the northern component, although the spectral index seems to stabilise at $\alpha=-0.3$, with the decreasing trend stalling between 56608.1 and 56609.1, just after the northern component first appears on MJD 56607.8. This may be suggestive of some particle acceleration at the shock giving rise to the northern component.

\subsubsection{Comparison to the X-ray behaviour}

As demonstrated by fig. 4 of Curran et al. (2015), the flare in the VLA and AMI light curves between MJD 56603 and 56620 took place during a period of very soft X-ray photon index, likely corresponding to either the soft-intermediate or soft X-ray spectral state. Indeed, a subsequent analysis of the X-ray spectral evolution by Zhang et al. (2015) suggests that XTE J1908+094 moved from an intermediate state on MJD 56599.5 to a soft state by the time of the following Swift/XRT observation on MJD 56604.9. Although the absence of X-ray timing information and the sparsity of the XRT coverage does not allow us to unambiguously determine the exact date of the state transition from the Swift data alone, the better-sampled MAXI data provide additional constraints. Plotting a hardness-intensity diagram from the publicly-available MAXI light curves $^{2}$ shows a clear softening between MJD 56598.5 and 56603.3 (Fig. 8).

We find that our inferred zero-separation date for the jet ejecta corresponds to the beginning of the spectral softening, making it plausible that the components did indeed move ballistically between the ejection date and our first VLBI detections. However, we cannot rule out some deceleration should the ejection have taken place during the latter part of the X-ray spectral softening. The sharp radio flare detected by AMI-LA on MJD 56601 occurs towards the end of the X-ray spectral softening, in the gap between the final intermediate state observation and the first detection of the soft state (as classified from the Swift/XRT data by Zhang et al. 2015). Given the unknown delay between ejection date and any internal shocks forming and becoming optically thin (Fender et al. 2009), we can only infer that the ejection event took place earlier than this. Finally, with the exception of

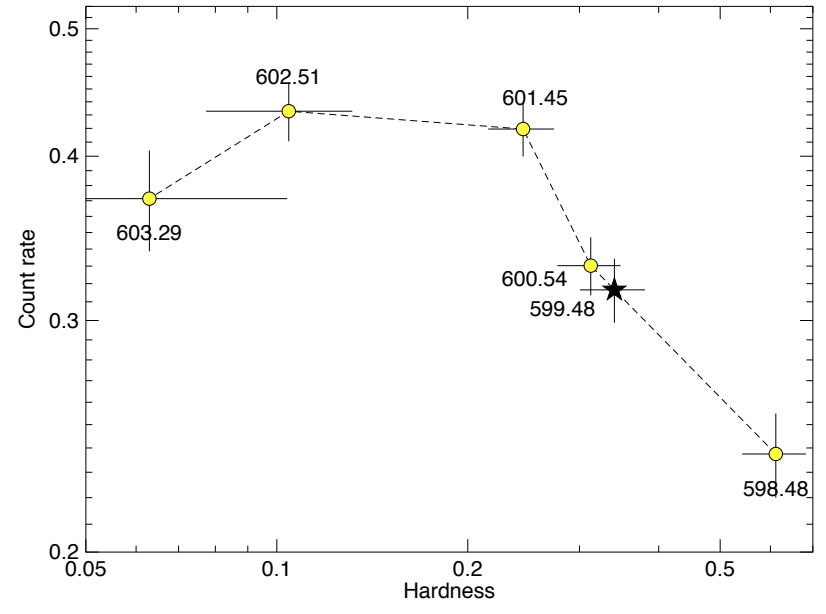

Figure 8. X-ray hardness-intensity diagram made from the publicly-available MAXI data. Hardness is the ratio of counts in the $6-20$ and $2-6 \mathrm{keV}$ bands, and intensity is the total $2-20 \mathrm{keV}$ count rate. The date of each observation is shown as MJD-56000. The black star shows the observation closest in time to the zeroseparation epoch determined in Section 3.1.

the first VLBA epoch, all our VLBI observations took place in the soft X-ray spectral state.

The hard X-ray flare detected by NuSTAR around MJD 56605.3 and lasting for $40 \mathrm{ks}$ (Zhang et al. 2015; Tao et al. 2015) occurred during the rise phase of the large radio flare, between our second and third VLBA observations (epochs B and C in Fig. 7). Although both Zhang et al. (2015) and Tao et al. (2015) suggested that the flare could have signified the ejection of new transient radio-emitting jet knots, our VLBI imaging shows no new jet component appearing at this time. The only clear radio changes that could potentially be associated with the hard X-ray flare are the slight flattening of the radio spectral index and the decrease in fractional polarization seen by the VLA between MJD 56605 and 56607, the development of the bright "core" in the southern jet component on MJD 56606.8, and the appearance of the northern component on MJD 56607.8. However, with the available data, we cannot unambiguously tie the hard X-ray flare to any of these events.

\subsubsection{Comparison to the 2002 outburst}

Rupen et al. (2002a) detected the radio counterpart of XTE J1908+094 during its 2002 outburst. The first detection was on March 21, and the source was detected through May 13 (Rupen et al. 2002b). The radio monitoring was relatively sparse, with ten observations over that 53-day period. This monitoring ${ }^{3}$ showed that the radio emission peaked on March 30, with non-detections on April 7 and 15, and the source reappearing as a resolved double on April 23 and May 1. On April 23, the two components had an angular separation of $\sim 0.3^{\prime \prime}$ and a position angle $-14 \pm 6^{\circ} \mathrm{E}$ of $\mathrm{N}$ (Fig. 9). While this position angle is very similar to that observed

3 http://www.aoc.nrao.edu/ mrupen/XRT/X1908+094/x1908+ 094.shtml

2 http://maxi.riken.jp/mxondem/ 


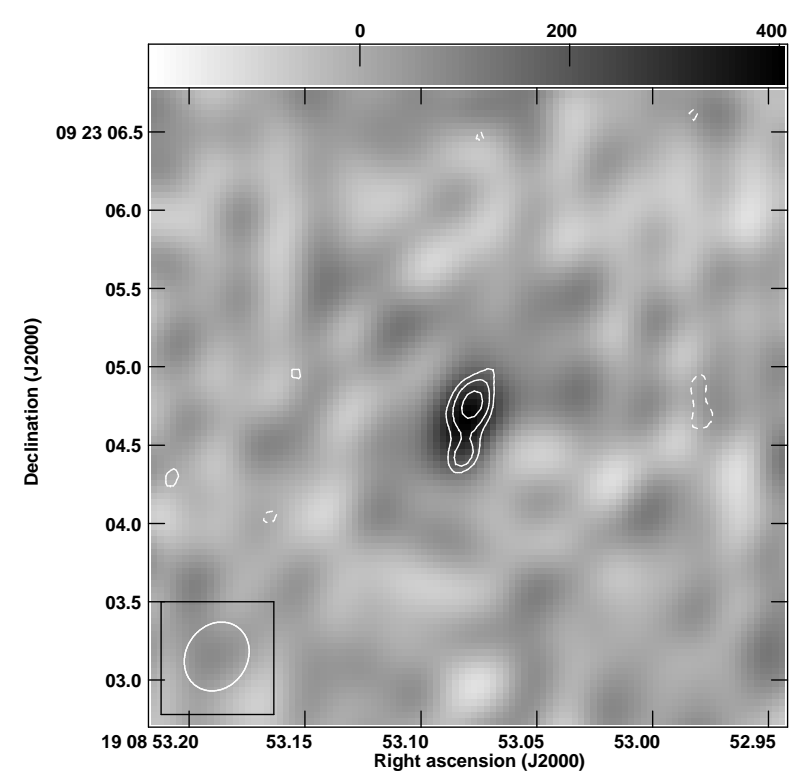

Figure 9. VLA image of XTE J1908+094, taken on 2002 April 23. Contours show the 8.4-GHz data and greyscale shows the 4.9$\mathrm{GHz}$ data. Contours are at levels of $\pm \sqrt{2}^{n}$ times the rms noise of

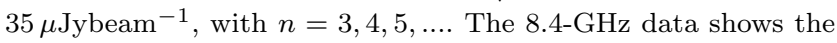
source to be resolved into two components separated by $326 \pm$ 35 mas along a position angle of $-14 \pm 6^{\circ} \mathrm{E}$ of $\mathrm{N}$. The overall radio spectrum is steep, with $\alpha=-0.8 \pm 0.4$. The VLA showed the source to be extended along the same position angle during the 2002 outburst as seen during the 2013 outburst, but with a significantly greater angular separation of the components.

in our VLBI data, their observations were conducted with the VLA in its A-configuration and thus had significantly lower spatial resolutions than ours (beam sizes of $0.2-0.3^{\prime \prime}$ at $8.46 \mathrm{GHz}$ ). This implies that the jet components reached significantly greater angular separations than seen in our 2013 observations. They also found the southern component to fade first, but the peak brightness detected in their images was from the northern component.

As in our 2013 data, several of the 2002 radio detections were made following a rebrightening during the soft state (as identified by Göğüs et al. 2004), which lasted from April 6 through June 4 . The peak radio flux of $1.8 \mathrm{mJy}_{\text {beam }}{ }^{-1}$ was detected on March 30, one week before the X-ray peak on April 6, and at the end of the hard X-ray state during the rise phase, as classified by Göğüş et al. (2004). Radio emission was not detected in the first soft-state observation on April 7 , but the source reappeared as a resolved double between 17 and 26 days after the onset of the soft state.

\subsection{Plausible scenarios}

We see significant discrepancies between the observed behaviour of XTE J1908+094 and the standard expectations of intrinsically symmetric transient jets that are ejected from the core at the state transition, propagate outwards and then fade away (e.g. Fender et al. 2004). These discrepancies include the delayed appearance of the northern component, the strong evolution of the brightness and morphology of the southern component, and the measured proper motions and relative flux densities of the two components. Possible scenarios that could explain the observations involve either absorption (either intrinsic or external to the source) that affects the observed flux density from one or both components, and the re-acceleration of particles downstream due to either internal or external shocks. Assuming an intrinsically symmetric ejection, we now assess the relative merits of these different explanations.

\subsubsection{Internal self-absorption}

The delayed appearance of the northern component could be due to its slower expansion, for instance due to it remaining confined (e.g. by magnetic fields or external pressure) for longer. The faster expansion of the southern component would enable it to become optically thin earlier, allowing us to see it appear and brighten in our VLBA images several days before the northern component ceased to be affected by the relevant absorption mechanism; likely either synchrotron self-absorption or free-free absorption. We consider this scenario to be unlikely, as at no stage did Curran et al. (2015) observe an inverted radio spectrum, and we saw no major change in the radio spectral index on the appearance of the northern component.

\subsubsection{External obscuration}

External absorption due to dense ambient material, an equatorial wind or even an an obscuring torus could mask the synchrotron radiation until the receding knot had moved out to a point where the optical depth was sufficiently low. While this could explain the apparent delayed appearance of the northern knot if that component was receding, this would be at odds with the higher measured proper motion of the northern component. We would need to invoke some sort of anisotropy in the surrounding medium that could slow the approaching southern component. Any such interaction with the surroundings would create a shock that would have been observed in the integrated radio light curves.

If the obscuration were due to free-free absorption, we can estimate the absorption coefficient (Verschuur \& Kellermann 1988) as

$k_{c} \cong \frac{0.08235 n_{e}}{\nu^{2.1} T_{\mathrm{e}}^{1.35}}$,

where $\nu$ is the frequency in $\mathrm{GHz}, n_{e}$ is the electron density in units of $\mathrm{cm}^{-3}$ and $k_{c}$ is in $\mathrm{pc}^{-1}$. If we assume the components appear when the optical depth $\left(k_{c} d s\right)$ reaches unity $(d s \sim$ 10 mas from the core) and $T_{\mathrm{e}}=10^{4} \mathrm{~K}$, then at $8.4 \mathrm{GHz}$, $n_{e} \approx 2.7 \times 10^{8} \mathrm{~cm}^{-3}$. This is comparable in density to the solar corona (Doschek et al. 1997; Warren \& Brooks 2009), but would need to stretch over tens of $\mathrm{AU}$, and would have a total mass of $10^{-5}-10^{-3} M_{\odot}$ (depending on the geometry). This seems unlikely, since the binary companion is suggested to be a main sequence star (Chaty et al. 2006). Thus, we do not favour this explanation.

\subsubsection{Internal shocks}

The components could instead be internal shocks that formed within the jets and propagated downstream (e.g. Fender et al. 2004; Jamil et al. 2010; Kaiser et al. 2000). However, to explain the higher proper motion, lower flux density, and delayed appearance of the northern component, 
we would again need to invoke asymmetries in the approaching and receding jets, making this explanation similarly unsatisfactory.

\subsubsection{External shocks}

The one explanation that could naturally explain the asymmetries in the northern and southern components is that we are observing the shocks created as the jets encounter a dense region of the ISM (possibly after reaching the edge of a cavity inflated by jets or accretion disc winds; see, e.g. Hao \& Zhang 2009). Different ISM densities on either side of the source could give rise to the asymmetry, with shockacceleration of particles at the interface then generating the observed radio emission.

The only previous cases in which lateral expansion of jet knots have been directly observed are the BH XRB XTE J1752-223 and the neutron star X-ray binary Sco X-1. In XTE J1752-223, Yang et al. (2010) found one of the ejecta to have expanded at $0.9 \pm 0.1 \mathrm{mas} \mathrm{d}^{-1}$, corresponding to $0.02 c$ at the claimed distance of $3.5 \mathrm{kpc}$ (Shaposhnikov et al. 2010). The ejecta were also seen to be decelerating, and were inferred to be interacting with the surrounding environment on scales of a few hundred mas (thousands of AU) downstream (Yang et al. 2010; Miller-Jones et al. 2011).

Fomalont et al. (2001) made intensive VLBI observations of Sco X-1, resolving the system into a central core and two downstream lobes that moved outwards in opposite directions at speeds varying from $0.32-0.58 c$. They found that each individual knot moved ballistically for many hours, although different knots had different inferred intrinsic speeds. During one epoch in 1998 February, they measured the expansion of the northeastern lobe, which was extended perpendicular to the jet direction and increased in size by a factor of 4.5 over $50 \mathrm{~min}$, implying an expansion velocity close to $c$. Fomalont et al. (2001) modelled the lobes as the working surfaces where the relativistic jets of Sco X-1 impacted the ambient medium. The electrons accelerated at the working surface then diffuse outwards along the radial direction at $0.57 c$ to form an extended lobe.

A similar interaction scenario could explain much of the behaviour seen in XTE J1908+094. The late-time radio flare corresponding to the appearance and evolution of the VLBI components could be explained as the time taken for the jets launched close to the black hole to propagate outwards and impact the ISM. The jets would have been launched at the hard-to-soft X-ray state transition (possibly giving rise to the short-lived radio flare on MJD 56601), and then decayed via adiabatic expansion to give the observed radio quenching. When the ejected material then ran into the external medium, it would have produced a shock at the working surface, observed as the VLBI ejecta that we monitored. The delayed appearance of the northern component would then be due to the different environmental densities on the two sides of the source.

The initial shock compression of the magnetic field at the working surface would give rise to the high degree of linear polarization seen on MJD 56605.0 (up to $13 \%$ at $7.45 \mathrm{GHz}$ and $10 \%$ at $5.25 \mathrm{GHz}$ ). The fractional polarization then drops to $1-2 \%$ on MJD 56607.0 as the electrons accelerated at the shock diffuse outwards and the magnetic field becomes less ordered, before recovering to $5 \%$ the following day as the northern component appears, giving rise to new shock compression. The gradual rotation of the EVPA between MJD 56604 and 56607 would be due to the diffusion of the accelerated electrons away from the shock-compressed magnetic field at the working surface. The shock acceleration at the working surface as the core of the southern component rebrightens on MJD 56606.8 could also explain the slight flattening of the radio spectrum seen between MJD 56605 and 56607, although the dominance of the halo component means that the impact of this new shock acceleration is only mild.

In this scenario, the brightness of the two components would be a reflection of both any intrinsic Doppler boosting and also the strength of the interaction with the ambient medium. Similarly, the hotspot advance speed would be dictated by the pressures in the shocked and unshocked regions (Fomalont et al. 2001), rather than by the intrinsic speed of the jets launched close to the black hole. Indeed, Fomalont et al. (2001) found a case where one of the hotspots clearly decelerated as it underwent a flare, due to forming a shock on interaction with the surrounding medium. Given the potential role of the environment in giving rise to the observed differences between the northern and southern components in XTE J1908+094, the constraint on $\beta \cos \theta$ derived from the proper motions of the northern and southern components may not be representative of the underlying, intrinsic jet parameters. Similarly, the derived core location may not be correct. Nonetheless, if we assume that the original ejection event was on or before the detection of the short-lived radio flare detected by AMI-LA, we still infer a low projected jet speed. The first detections of the northern and southern components are separated by 25 mas and four days, implying a maximum projected jet speed of $0.29 c(d / 8 \mathrm{kpc})$.

Under this scenario, it is interesting to consider the 40ks NuSTAR flare reported by Zhang et al. (2015) and Tao et al. (2015) (see Section 4.1.3). If this was indeed a flare from the corona, there is no corresponding signature in the VLBI images until either the rebrightening of the core of the southern component on MJD 56606.8, or the development of the northern component on MJD 56607.8. While these associations with downstream events are tentative at best, we can determine the implications of such a scenario, which would then give a time difference of 1.5-2.5 days between the $\mathrm{X}$-ray flare and any radio signature. The southern core on MJD 56606.8 is separated from the inferred binary location by $12.5 \pm 3.3$ mas, and the northern component on MJD 56607.8 by $18.9 \pm 3.6$ mas. This would therefore imply proper motions of $\sim 8 \pm 2$ mas d if the energy were to propagate directly downstream from the core to the working surfaces, for a projected speed (in the plane of the sky) of $0.3-0.4 c$. This would be similar to the scenario observed in Sco X1 , where the energy was transferred from the core to the lobes at $>0.95 c$, much faster than the advance speed of the hotspot (Fomalont et al. 2001).

\subsection{Energetics}

The southern component reached its peak brightness on MJD 56606.8, when it had a total integrated flux of $15.6 \pm 0.8 \mathrm{mJy}$, and a Gaussian full-width at half maximum $(\mathrm{FWHM}=2 \sqrt{2 \ln 2} \sigma)$ of 14 mas. Assuming a representative distance of $8 \mathrm{kpc}$ and a spherical geometry, this would im- 
ply an emitting volume of $2 \times 10^{45} \mathrm{~cm}^{-3}$. We performed a standard minimum energy calculation (e.g. Pacholczyk 1970; Longair 1994; Rohlfs \& Wilson 1996) by assuming an underlying electron energy distribution index $p=2.2$ (corresponding to a spectral index of -0.6 when fully optically thin), a spectrum extending between at least $10^{7}$ and $10^{11} \mathrm{~Hz}$, and as much energy in protons as there is in relativistic electrons. The minimum energy and corresponding minimum magnetic field strength were determined via

$E_{\min }=\frac{7}{6 \mu_{0}} V^{3 / 7}\left[\frac{3 \mu_{0}}{2} G(\alpha) \eta L_{\nu}\right]^{4 / 7}$

$B_{\min }=\left[\frac{3 \mu_{0}}{2} \frac{G(\alpha) \eta L_{\nu}}{V}\right]^{2 / 7}$,

where $G(\alpha)$ is a constant that loosely depends on $\alpha, \nu_{\text {min }}$ and $\nu_{\max }$. We find a minimum energy of $E_{\min }=5 \times 10^{41} \mathrm{erg}$, and a minimum energy field of $B_{\mathrm{min}}=50 \mathrm{mG}$.

In comparison, both GRS 1915+105 and XTE J1748-288 were found to have had ejection events with minimum energies of a few times $10^{42}$ erg (Fender et al. 1999; Brocksopp et al. 2007). In contrast, a detailed study of a sequence of ejection events in XTE J1752-223 showed minimum energies for the different events ranging from $10^{41}-7 \times 10^{42} \mathrm{erg}$, showing that different ejection events in an individual source can vary greatly in energy (Brocksopp et al. 2013). Thus, the minimum energy derived for the main radio flare observed in XTE J1908+094 was on the low end of what has been seen from other BH XRB jets, but by no means an outlier relative to the overall distribution.

Knowing the distance downstream at which the jets slow down and form hotspots, we can use the formalism of Heinz (2002) to constrain the environmental density, which is given by

$n \sim \frac{E_{44}}{\Gamma_{5}^{2} \theta_{5}^{2} d_{16}^{3}} \mathrm{~cm}^{-3}$,

where $E_{44}$ is the energy in the jets in units of $10^{44} \mathrm{erg}$, the jet Lorentz factor is $\Gamma=5 \Gamma_{5}$, the knot opening angle is $\theta=5^{\circ} \theta_{5}$, and the jet decelerates due to its interaction with the surroundings at a distance $10^{16} d_{16} \mathrm{~cm}$. We assume a canonical Lorentz factor of $\Gamma=2$, an opening angle of $\theta=58^{\circ}$ (as derived from the size and downstream distance of the southern lobe on MJD 56606.8) and a distance downstream from the binary system of $d=0.15 \times 10^{16} \mathrm{~cm}$. This implies a density $n=13 E_{44} \mathrm{~cm}^{-3}$. Given the minimum energy determined above, this would place a lower limit on the density of $n \geq 0.07 \mathrm{~cm}^{-3}$, such that the jets would not need to be far out of equipartition in order for the local ISM density to be comparable to the canonical value of $1 \mathrm{~cm}^{-3}$. If (as seems likely) the actual jet opening angle is smaller (i.e. the measured size instead reflects the lateral expansion of the lobe, and not of the jet itself), the inferred density would be higher still. This is in stark contrast to GRS 1915+105 and GROJ1655-40, which Heinz (2002) found to be located in significantly more underdense environments, with $n<10^{-3} \mathrm{~cm}^{-3}$.

Hao \& Zhang (2009) modelled the jet-ISM interactions in XTE J1550-564 and H1743-322 by assuming that the observed emission was produced at the reverse shock generated when the jets, having propagated outwards through a low-density cavity, encountered the denser, surrounding ISM. They inferred similarly low ISM densities of $\sim 3 \times 10^{-3} \mathrm{~cm}^{-3}$ around H1743-322 and the eastern jet of XTE J1550-564. However, the latter source was found to lie in a very inhomogeneous environment, with the western jet running into a higher-density medium with $n=0.12 \mathrm{~cm}^{-3}$. Without more detailed knowledge of the core location or the ejection time (which we inferred in Section 3.1 by assuming ballistic motions for our components and extrapolating back to zero separation), we do not have sufficient constraints to apply such a model to XTE J1908+094. However, should our target be located in a low-density cavity (as hypothesised to exist around all microquasar systems by Hao \& Zhang 2009), the extent of the cavity must be significantly lower than in XTE J1550-564 or H1743-322, given that the jet-ISM interaction was seen to occur on scales of a few tens of milliarcseconds rather than several arcseconds. This would imply that the jets or accretion disc winds responsible for inflating the cavity were significantly weaker in XTE J1908+094 (and also in XTE J1752-223, where jetISM interactions were seen on scales of a few hundred milliarcseconds), or that the outflows responsible for creating the cavity had been active for less time.

The different distances to which the jets propagated during the 2002 and 2013 outbursts would seem to argue against a single, unchanging, large-scale cavity around XTE J1908+094, as the jet-ISM interactions occurred much closer to the central binary system in 2013 than in 2002 . Nonetheless, the 2002 outburst also seems consistent with an external shock scenario, with the system going through an initial flare, followed by a period of non-detections, and finally a rebrightening during which time the resolved ejecta were detected (see Section 4.1.4). As in 2013, the downstream rebrightening of these resolved ejecta would seem to argue for interactions between the jet and the ISM. Given the relatively sparse time sampling, we cannot determine whether the southern interaction region ever got as bright as its northern counterpart prior to April 23 (the date of the image in Fig. 9). Regardless, the appearance of the knots further downstream in 2002 suggests a difference in the properties of either the jets or the environment.

In the absence of a large-scale cavity, we can instead explore the uniform density scenario of Heinz (2002). Had there been no significant change in the external density between 2002 and 2013, Equation (7) would suggest that despite comparable peak X-ray fluxes (Curran et al. 2015), the jets of XTE J1908+094 were more energetic in 2002 than in 2013 , such that they could propagate out to a larger distance. With the two components in Fig. 9 being separated by $326 \pm 35$ mas, the most distant component must be at least 163 mas from the core, implying a downstream distance $d_{16}=2.0$. Therefore, if the environmental density, jet Lorentz factor and opening angle were unchanged, $E_{44}$ would need to have been 2000 times as great in 2002 as in 2013. Given the comparable peak X-ray fluxes, this seems implausible. Either the properties of the environment were different (e.g. a decrease in the density on moving further away from the binary system, since Equation (7) assumes a uniform density), or the jet Lorentz factor and/or opening angle were smaller in 2002 . 


\subsection{Comparison to other systems}

Laterally expanding jets are a rare phenomenon in low-mass BH XRBs (Miller-Jones et al. 2006), with the only example to date having been seen in XTE J1752-223 (Yang et al. 2010), whose jet was also thought to have been interacting with the ISM. Similar expanding working surfaces were also seen in the neutron star XRB Sco X-1 (Fomalont et al. 2001). These were regenerated during multiple individual ejection events, always moving ballistically, and appearing at the same position angle with respect to the central source, in the same way that XTE J1908+094 showed jets along the same position angle in both 2002 and 2013. However, the working surfaces moved outwards faster in both XTE J1752-223 and Sco X-1 than in XTE J1908+094 (despite the lower calculated minimum energy of $6 \times 10^{39} \mathrm{erg}$ in Sco X-1).

A third system showing laterally-expanding outflows was the accreting source CI Cam during its 1998 outburst. VLBI observations by Mioduszewski \& Rupen (2004) suggested that the compact object ejected strong jets that were smothered by a dense circumstellar medium, leading to a much less collimated outflow than typically observed from $\mathrm{X}$-ray binary jets. However, the recent Gaia distance measurement to this system has suggested that it is an accreting white dwarf rather than a neutron star XRB (Wijngaarden et al. 2016; Barsukova et al. 2006).

While lateral expansion appears to be rare, the deceleration of jets as they interact with the ISM has been seen in a number of sources in recent years, over a wide range of scales. The jets in XTE J1752-223 decelerated on a scale of a few hundred mas (Yang et al. 2010, 2011; Miller-Jones et al. 2011), on a timescale of a few tens of days following the flare. On the other hand, the jets of XTE J1550-564 and H1743-322 were both seen to decelerate significantly further downstream, at distances of several arcseconds, and hundreds of days following the original outburst (Corbel et al. 2002; Kaaret et al. 2003; Corbel et al. 2005). According to the formalism of Heinz (2002), this would imply either smaller opening angles, lower environmental densities, or more energetic jets in the latter two cases. Alternatively, should these systems in fact be surrounded by low-density cavities inflated by accretion disc winds or jets (as suggested by Hao \& Zhang 2009), the cavities must be significantly smaller, and hence the strength of the outflows correspondingly weaker in XTE J1752-223 and XTE J1908+094.

\section{CONCLUSIONS}

We have presented a rare example of lateral expansion of the jets from a BH XRB. Our high-resolution VLBI monitoring of the 2013 outburst of XTE J1908+094 has shown the development of asymmetric, resolved, expanding ejecta following a hard to soft state transition. These ejecta move in opposite directions with proper motions of $2-3$ mas day $^{-1}$, implying relatively low jet speeds of $<0.3 c(d / 8 \mathrm{kpc})$. The ejecta appear to expand isotropically at a rate of $\sim 3$ mas day $^{-1}$. We interpret these jet components as the working surfaces where the jets ejected at the hard-to-soft state transition impacted the ISM, creating a moving shock front that accelerated particles, which subsequently diffused outwards over time.
Minimum energy calculations showed that the jet energy was at the low end of the observed distribution of BH XRB jets, and suggested that the surrounding ISM was denser than seen around other systems such as GRS 1915+105 and GRO J1655-40.

Comparison with the photometric and polarimetric data from integrated radio light curves shows that the jetISM interaction created a long-lived radio flare, whose evolution was linked to the appearance and evolution of the two resolved VLBI components. This long-lived flare reached flux densities comparable to the rapid initial flare detected by AMI-LA at the state transition, and suggests that care should be taken in conducting jet-disc coupling studies without accompanying high angular resolution images. Integrated light curves alone are unable to distinguish between internal and external shock scenarios, such that VLBI monitoring is required to accurately interpret contemporaneous radio and X-ray phenomenology.

\section{ACKNOWLEDGMENTS}

We are deeply indebted to Peter Curran, who drove much of this work and was responsible for a significant amount of the data collation and interpretation before his death. His contributions to the field will be greatly missed by his many colleagues. The National Radio Astronomy Observatory is a facility of the National Science Foundation operated under cooperative agreement by Associated Universities, Inc. The European VLBI Network is a joint facility of independent European, African, Asian, and North American radio astronomy institutes. Scientific results from data presented in this publication are derived from the following EVN project codes: RR007 and RR009. This work made use of the Swinburne University of Technology software correlator, developed as part of the Australian Major National Research Facilities Programme and operated under licence. APR acknowledges funding via an EU Marie Curie Intra-European Fellowship under contract no. 2012-331977. The work was also supported by ERC grant 267697 "4 PI SKY: Extreme Astrophysics with Revolutionary Radio Telescopes". JCAMJ is supported by an Australian Research Council Future Fellowship (FT140101082). This work was supported by Australian Research Council grant DP120102393. DA acknowledges support from the Royal Society.

\section{REFERENCES}

Brocksopp C., Miller-Jones J. C. A., Fender R. P., Stappers B. W., 2007, MNRAS, 378, 1111

Brocksopp C., Corbel S., Tzioumis A., Broderick J. W., Rodriguez J., Yang J., Fender R. P., Paragi Z., 2013, MNRAS, 432, 931

Barsukova E. A., Borisov N. V., Burenkov A. N., Goranskii V. P., Klochkova V. G., Metlova N. V., 2006, Astronomy Reports, 50,664

Belloni, T. M. 2010, Lecture Notes in Physics, Berlin Springer Verlag, 794, 53

Chaty, S., Mignani, R. P., \& Israel, G. L. 2002, MNRAS, 337, L23

Chaty, S., Mignani, R. P., \& Israel, G. L. 2006, MNRAS, 365, 1387

Corbel S., Fender R. P., Tzioumis A. K., Tomsick J. A., Orosz J. A., Miller J. M., Wijnands R., Kaaret P. 2002, Science, 298, 196 
Corbel S., Fender R. P., Tzioumis A. K., Tomsick J. A., Orosz J. A., Miller J. M., Wijnands R., Kaaret P. 2003, NewAR, 47, 477

Corbel S., Kaaret P., Fender R. P., Tzioumis A. K., Tomsick J. A., Orosz J. A. 2005, ApJ, 632, 504

Corbel, S. et al. 2013, MNRAS, 431, L107

Coriat M., Tzioumis T., Corbel S., Fender R., 2013, The Astronomer's Telegram, 5575, 1

Curran P. A., et al., 2014, MNRAS, 437, 3265

Curran, P. A. et al. 2015, MNRAS, 451, 3975

Deller A. T., Tingay S. J., Bailes M., West C. 2007, PASP, 119, 318

Dhawan, V., Mirabel, I. F., \& Rodríguez, L. F. 2000, ApJ, 543, 373

Doschek G. A., Warren H. P., Laming J. M., Mariska J. T., Wilhelm K., Lemaire P., Schü e, U., Moran T. G., 1997, ApJ, 482, L109

Fender R. P., Garrington S. T., McKay D. J., Muxlow T. W. B., Pooley G. G., Spencer R. E., Stirling A. M., Waltman E. B. 1999, MNRAS, 304, 865

Fender, R. 2006, in Lewin W. H. G., van der Klis M., eds, Cambridge Astrophysics Series, No. 39, Compact Stellar X-ray Sources. Cambridge Univ. Press, Cambridge, p. 381

Fender, R., Belloni, T., \& Gallo, E. 2004, MNRAS, 355, 1105

Fender R. P., Homan J., Belloni T. M., 2009, MNRAS, 396, 1370

Feroci, M., Reboa, L., \& BEPPOSAX Team 2002, IAUC., 7861, 2

Fomalont, E. B., Geldzahler, B. J., \& Bradshaw, C. F. 2001, ApJ, 558,283

Fomalont E. B., Geldzahler B. J., Bradshaw C. F., 2001, ApJ, 553, L27

Gallo E., Fender R., Kaiser C., Russell D., Morganti R., Oosterloo T., Heinz S. 2005, Nat, 436, 819

Garnavich, P., Quinn, J., \& Callanan, P. 2002, IAUC., 7877, 4

Göğüş, E. et al., 2004, ApJ, 609, 977

Greisen E. W., 2003, in Heck A., ed., Information Handling in Astronomy: Historical Vistas. Kluwer, Dordrecht, p. 109

Hao J. F., Zhang S. N., 2009, ApJ, 702, 1648

Heinz, S. 2002, A\&A, 388, L40

Heinz, S., Grimm, H. J., Sunyaev, R. A., \& Fender, R. P. 2008, ApJ, 686, 1145-1154

Hjellming, R. M., \& Rupen, M. P. 1995, Nat, 375, 464

in 't Zand, J. J. M., Capalbi, M., \& Perri, M. 2002a, IAUC., 7873, 1

in't Zand, J. J. M., Miller, J. M., Oosterbroek, T., \& Parmar, A. N. 2002b, A\&A, 394, 553

Jamil, O., Fender, R. P., \& Kaiser, C. R. 2010, MNRAS, 401, 394

Jonker P. G., Gallo E., Dhawan V., Rupen M., Fender R. P., Dubus G. 2004, MNRAS, 351, 1359

Kaaret P., Corbel S., Tomsick J. A., Fender R., Miller J. M., Orosz J. A., Tzioumis A. K., Wijnands R., 2003, ApJ, 582, 945

Kaiser, C. R., Sunyaev, R., \& Spruit, H. C. 2000, A\&A, 356, 975

Kalemci E., Dinç, T., Tomsick J. A., Buxton M. M., Bailyn C. D., Chun Y. Y., 2013, ApJ, 779, 95

Kaiser, C. R. 2006, MNRAS, 367, 1083

Keimpema, A., Kettenis, M. M., Pogrebenko, S. V., et al. 2015, Experimental Astronomy, 39, 259

Kettenis, M., van Langevelde, H. J., Reynolds, C., \& Cotton, B. 2006, in Gabriel C., Arviset C., Ponz D., Solano E., eds, ASP Conf. Ser. Vol. 351, Astronomical Data Analysis Software and Systems XV. Astron. Soc. Pac., San Francisco, p. 497

Krimm, H. A., et al. 2013, The Astronomer's Telegram, 5523, 1

Krimm, H. A., Kennea, J. A., \& Holland, S. T. 2013, The Astronomer's Telegram, 5529, 1

Kubota A., Makishima K., Ebisawa K., 2001, ApJ, 560, L147

Ling Z., Zhang S. N., Tang S., 2009, ApJ, 695, 1111

Longair, M. S. 1994, High energy astrophysics. Vol.2: Stars, the galaxy and the interstellar medium, 2nd Ed. Cambridge: Cambridge University Press

Maccarone T. J., 2003, A\&A, 409, 697

McHardy, I. M., Koerding, E., Knigge, C., Uttley, P., \& Fender, R. P. 2006, Nat, 444, 730

Miller J. M., Reynolds C. S., Fabian A. C., Miniutti G., Gallo L. C., 2009, ApJ, 697, 900

Miller-Jones J. C. A., Blundell K. M., Rupen M. P., Mioduszewski A. J., Duffy P., Beasley A. J. 2004, ApJ, 600, 368

Miller-Jones J. C. A., Blundell K. M., Duffy P., 2004b, ApJ, 603, L21

Miller-Jones, J. C. A., Fender, R. P., \& Nakar, E. 2006, MNRAS, 367,1432

Miller-Jones J. C. A., Rupen M. P., Fender R. P., Rushton A., Pooley G. G., Spencer R. E. 2007, MNRAS, 375, 1087

Miller-Jones J. C. A., Jonker P. G., Ratti E. M., Torres M. A. P., Brocksopp C., Yang J., Morrell N. I. 2011, MNRAS, 415, 306

Miller-Jones J. C. A. et al., 2012, MNRAS, 421, 468

Miller-Jones J. C. A., Sivakoff G. R., Krimm H. A., 2013, The Astronomer's Telegram, 5530, 1

Mioduszewski A. J., Rupen M. P., Hjellming R. M., Pooley G. G., Waltman E. B. 2001, ApJ, 553, 766

Mioduszewski, A. J., \& Rupen, M. P. 2004, ApJ, 615, 432

Mirabel, I. F., Rodriguez, L. F., Cordier, B., Paul, J., \& Lebrun, F. 1992, Nat, 358, 215

Mirabel, I. F., \& Rodríguez, L. F. 1994, Nat, 371, 46

Mirabel, I. F., \& Rodriguez, L. F. 2002, Sky and Telescope, 103, 32

Mirabel, I. F., Dijkstra, M., Laurent, P., Loeb, A., \& Pritchard, J. R. 2011, A\&A, 528, AA149

Pacholczyk A. G., 1970, Radio Astrophysics. Freeman \& Co., San Francisco

Pakull, M. W., Soria, R., \& Motch, C. 2010, Nat, 466, 209

Reid M. J., McClintock J. E., Steiner J. F., Steeghs D., Remillard R. A., Dhawan V., Narayan R. 2014, ApJ, 796, 2

Reynolds, C., Paragi, Z., \& Garrett, M. 2002, arXiv:astro$\mathrm{ph} / 0205118$

Rodriguez, L. F., Mirabel, I. F., \& Marti, J. 1992, ApJL, 401, L15

Rohlfs K., Wilson T. L., 1996, Tools of Radio Astronomy (Astronomy and Astrophysics Library). Springer-Verlag, Berlin, p. 190

Rupen, M. P., Dhawan, V., \& Mioduszewski, A. J. 2002a, IAUC., 7874,1

Rupen, M. P., Mioduszewski, A. J., \& Dhawan, V. 2002b, IAUC., 8029, 2

Rushton, A., Spencer, R. E., Pooley, G., \& Trushkin, S. 2010, MNRAS, 401, 2611

Rushton, A., Spencer, R., Fender, R., \& Pooley, G. 2010, A\&A, 524, A29

Rushton A. P., Fender R., Anderson G., Staley T., Rumsey C., Titterington D., 2013a, The Astronomer's Telegram, 5532, 1

Rushton A. P., Fender R., Anderson G., Staley T., Rumsey C., Titterington D., 2013b, The Astronomer's Telegram, 5551, 1

Russell, D. M., Fender, R. P., Gallo, E., \& Kaiser, C. R. 2007, MNRAS, 376, 1341

Sell, P. H., Heinz, S., Richards, E., et al. 2015, MNRAS, 446, 3579

Shaposhnikov N., Markwardt C., Swank J., Krimm H., 2010, ApJ, 723,1817

Shepherd M. C., in Hunt G., Payne H. E., eds, ASP Conf. Ser. Vol. 125, Astronomical Data Analysis Software and Systems VI. Astron. Soc. Pac., San Francisco, p. 77

Stirling A. M., Spencer R. E., de la Force C. J., Garrett M. A., Fender R. P., Ogley R. N. 2001, MNRAS, 327, 1273

Szomoru, A. 2008, The role of VLBI in the Golden Age for Radio Astronomy, 40

Tao L. et al., 2015, ApJ, 811, 51

Tingay S. J. et al., 1995, Nat, 374, 141

Verschuur G. L., Kellermann K. I., eds, 1988, Galactic and Ex- 
tragalactic Radio Astronomy, 2nd edn. Springer-Verlag, New York

Wagner, R. M., \& Starrfield, S. 2002, The Astronomer's Telegram, 86,1

Warren H. P., Brooks D. H., 2009, ApJ, 700, 762

Wiersema, K., Russell, D. M., Degenaar, N., et al. 2009, MNRAS, 397, L6

Wijngaarden M. J. P., Gourdji K., Oostrum L. C., Henrichs H. F., 2016, The Astronomer's Telegram, 9634, 1

Woods P. M., Kouveliotou C., Finger M. H., Gogus E., Swank J., Markwardt C., Strohmayer T. 2002, IAUC., 7856, 1

Yang J., Brocksopp C., Corbel S., Paragi Z., Tzioumis T., Fender R. P., 2010, MNRAS, 409, L64

Yang J., Paragi Z., Corbel S., Gurvits L. I., Campbell R. M., Brocksopp C. 2011, MNRAS, 418, L25

Yoon, D., Morsony, B., Heinz, S., et al. 2011, ApJ, 742, 25

Zhang L., Chen L., Qu J.-l., Bu Q.-c., Zhang W., 2015, ApJ, 813, 90

Zwart, J. T. L. et al. 2008, MNRAS, 391, 1545 